\title{
Recent advances in quartz enhanced photoacoustic sensing
}

\author{
Pietro Patimisco, ${ }^{1,2}$ Angelo Sampaolo, ${ }^{1,2}$ Lei Dong, $^{3, a)}$ Frank K. Tittel, ${ }^{2}$ \\ and Vincenzo Spagnolo ${ }^{1,2, a)}$ \\ ${ }_{1}^{1}$ PolySense Lab - Dipartimento Interateneo di Fisica, Università degli Studi di Bari and Politecnico di Bari, \\ CNR-IFN BARI, Via Amendola 173, Bari 70126, Italy \\ ${ }^{2}$ Department of Electrical and Computer Engineering, Rice University, Houston, Texas 77005, USA \\ ${ }^{3}$ State Key Laboratory of Quantum Optics and Quantum Optics Devices, Institute of Laser Spectroscopy, \\ Shanxi University, Taiyuan 030006, China
}

(Received 13 November 2017; accepted 13 February 2018; published online 1 March 2018)

\begin{abstract}
This review aims to discuss the latest advancements in quartz-enhanced photoacoustic spectroscopy (QEPAS) based trace-gas sensing. Starting from the QEPAS basic physical principles, the most used QEPAS configurations will be described. This is followed by a detailed theoretical analysis and experimental study regarding the influence of quartz tuning forks (QTFs) geometry on their optoacoustic transducer performance. Furthermore, an overview of the latest developments in QEPAS trace-gas sensor technology employing custom QTFs will be reported. Results obtained by exploiting novel micro-resonator configurations, capable of increasing the QEPAS signal-to-noise ratio by more than two orders of magnitude and the utilization of QTF overtone flexural modes for QEPAS based sensing will be presented. A comparison of the QEPAS performance of different spectrophone configurations is reported based upon signal-to-noise ratio. Finally, a novel QEPAS approach allowing simultaneous dual-gas detection will be described. Published by AIP Publishing.

https://doi.org/10.1063/1.5013612
\end{abstract}

\section{TABLE OF CONTENTS}

I. INTRODUCTION

II. QUARTZ-ENHANCED PHOTOACOUSTIC

SPECTROSCOPY

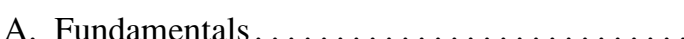

B. Fiber-based QEPAS

C. Modulation cancellation method (MOCAM)

D. Beat frequency (BF) quartz-enhanced photoacoustic spectroscopy (BF-QEPAS) ...

E. Intracavity QEPAS $\ldots \ldots \ldots \ldots \ldots \ldots$

F. QEPAS configuration based on dual micro-resonators and dual quartz tuning forks (QTFs) . . . . . . . . . . . . . .

III. QEPAS WITH CUSTOM QTFS $\ldots \ldots \ldots \ldots \ldots$

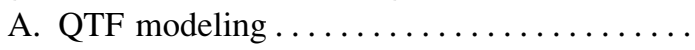

B. Custom QTFs design and characterization ..

C. QEPAS in the $\mathrm{THz}$ range $\ldots \ldots \ldots \ldots \ldots$

D. Single-tube micro-resonator systems ......

E. QEPAS with QTF operating in the first overtone flexural mode.............

F. Single-tube micro-resonator coupled with QTF operating at the first overtone flexural

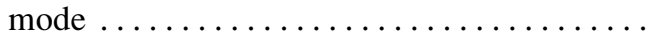

G. Double antinode excited quartz-enhanced photoacoustic spectrophone...........

1 2

2

3

4

4 5

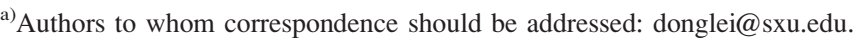
cn and vincenzoluigi.spagnolo@poliba.it
}

H. Simultaneous dual-gas detection.......... IV. CONCLUSIONS AND OUTLOOK...........

\section{INTRODUCTION}

Trace gas detection has long played an important role in developing and improving the technology and the solutions employed for gas sensing techniques. Many different types of gas sensors for a wide range of applications have been proposed. ${ }^{1-6}$ Part of these sensors are meant for the health and safety monitoring purposes. ${ }^{6}$ Hazardous toxic gases and air pollutants, such as methane and other hydrocarbons, which are considered to be environmental threats, exist in the atmosphere at part-per-million (ppm) concentration levels and below. Gas concentration assessment and control has become an important global issue to which new technologies can introduce innovative ways to develop sensitive and selective sensor systems. A wide range of non-optical gas detection approaches have been developed, employing laboratory analytical equipment, semiconductor gas sensors, and electrochemical devices. ${ }^{7}$ Optical methods for gas sensing can be either low-cost (but with limited detection sensitivity) chemical sensors and high-performance (but costly) laboratory equipment (such as gas chromatographs). Optical sensors can offer higher sensitivity, selectivity, and long-term stability with respect to other methods, 8,9 with advantages, such as a much longer lifetime and shorter response time, which enables real-time and in situ detection. Optical gas sensors are mainly based on light absorption based on the Lambert-Beer's law. The most commonly used techniques 
include non-dispersive infra-red (NDIR), Tunable Diode Laser Spectroscopy (TDLS), multi-pass or cavity enhanced spectroscopy, and Photoacoustic Spectroscopy (PAS). ${ }^{1,2}$ Photoacoustic spectroscopy is one of the most sensitive optical detection schemes. It is based on the detection of acoustic waves, which results from the absorption of light in a specific targeted gas. Light absorbed excites a fraction of the ground-state molecular population into higher energy levels. These excited states subsequently relax through a combination of radiative and non-radiative pathways. The nonradiative component ultimately generates heat in the localized region of the excitation light beam and a pressure wave propagates from the source, which is then detected by a microphone. The targeted gas is enclosed inside a resonant acoustic cell. The quality factor $\mathrm{Q}$ and the resonance frequency typically fall in the ranges of $40-200$ and $1-4 \mathrm{kHz}$, respectively. Photoacoustic detection is unique since it is a direct monitor of the nonradiative relaxation channel and, hence, complements absorption spectroscopic techniques. Furthermore, PAS does not require an optical detector and is wavelength independent. Other advantages include small size, large linear dynamic range [from few $\%$ to part-per-trillion (ppt) concentration range], and long-term stability. ${ }^{2}$ These advantages make the PAS technology competitive with, and in many cases, preferred to, other trace gas sensing methods. A significant improvement of the PAS technique was made by replacing the microphone with a high-Q piezoelectric quartz tuning fork (QTF) with a resonance frequency close to $32.7 \mathrm{kHz}$. This frequency refers to the plane flexural mode in which the QTF prongs move in opposite directions. This mode is piezoelectrically active (symmetric mode) and has a Q-value of 100000 or higher when it is encapsulated in vacuum and $>10000$ at normal atmospheric pressure. QTF-based PAS is referred to as quartz-enhanced photoacoustic spectroscopy or QEPAS. This review is aimed to discuss all advancements in QEPAS technique, since its invention in 2002. The review is organized in two sections. In Sec. I, fundamentals of QEPAS are described and different QEPAS configurations employing a $32.7 \mathrm{kHz}-\mathrm{QTF}$ are presented. In Sec. II, advances in QEPAS resulting from the employment of custom-made QTFs are reviewed, such as the extension of the QEPAS technique into the terahertz spectral range, the possibility of simultaneous-dual gas detection, and the acoustic coupling of the QTF with a micro-resonator tube, capable of amplifying the QEPAS signal-to-noise ratio by more than two orders of magnitude.

\section{QUARTZ-ENHANCED PHOTOACOUSTIC SPECTROSCOPY}

\section{A. Fundamentals}

QEPAS represents an alternative approach of traditional photoacoustic detection of gas traces. The basic idea of QEPAS is to accumulate the acoustic energy in a sharply resonant acoustic transducer, avoiding the use of traditional gas-filled photoacoustic cells, thereby removing restrictions imposed on the gas cell design by the acoustic resonance conditions. ${ }^{10}$ The transducer is positioned in the acoustic near-field zone of the laser excitation beam and the cell only serves to separate the target sample from the surrounding environment and fix and control its pressure. Conventional microphones are designed to have flat frequency response and are not suitable, whereas QTFs are perfect candidates to detect weak photoacoustic excitation, since they are characterized by a sharp resonant acoustic profile. QTFs operating in the few $\mathrm{kHz}$ range can be used, since the energy transfer processes in gases occur on a $\mu$ s time scale ${ }^{11}$ and the PAS signal decreases at higher frequencies. The most convenient is the QTF used in electronic timing devices, such as clocks and smart-phones as frequency standards, with a resonance frequency of $32768\left(2^{15}\right) \mathrm{Hz}$ in vacuum. A $32 \mathrm{kHz}-\mathrm{QTF}$ has been the only QTF employed in QEPAS sensor systems for the first ten years after the invention of this technique in 2002. ${ }^{12}$ Merits of QEPAS include a simple design, no requirement for a spectrally selective optical component, applicability over a wide range of pressure, including atmospheric pressure, capability to analyze trace-gas samples as low as few $\mathrm{cm}^{3}$ in volume, and immunity to external acoustic noise. A QTF has an acoustic quadrupole geometry, providing good environmental noise immunity. The only way to cause QTF to vibrate via the photoacoustic effect is to produce sound waves from an acoustic source located between the two QTF prongs. The standard way to realize such a condition is to focus the excitation laser beam through the gap between the prongs on the QTF crystal plane. Similar to PAS based sensing systems, the detection sensitivity of QEPAS sensor systems are proportional to the exciting laser optical power. ${ }^{2,12,13}$ The detected photoacoustic signal can be expressed in the same way as for PAS ${ }^{10}$

$$
S \propto \frac{Q \cdot P_{L} \cdot \alpha}{f},
$$

where $f$ is the QTF resonance frequency, $Q$ its quality factor, $\alpha$ is the gas target absorption coefficient, and $P_{L}$ is the laser power. Thus, the performance of QEPAS-based sensors can benefit from the consistently improved output powers of commercially available near infrared, mid-infrared lasers, and far infrared lasers due to the technology developments by the semiconductor industry.

Significant enhancements of the QEPAS signal-to-noise ratio have also been obtained as a result of the implementation of micro-resonators $(\mathrm{mR})$. A $\mathrm{mR}$ is formed by one or two thin tubes and the QTF is positioned either between (onbeam QEPAS ${ }^{14-17,20}$ ) or adjacent to the tube(s) (off-beam QEPAS $^{18,19,21-24}$ ) to probe the acoustic vibration excited in the gas contained inside the tubes. A sub system composed of the QTF and the mR is called a spectrophone or acoustic detection module (ADM). In both on-beam or off-beam sensor configurations, it is critical that the photoacoustic exciting radiation does not hit the $\mathrm{ADM}$, as otherwise an undesirable background that can be several times larger than the QTF thermal noise level arises, with a shifting fringe-like interference pattern shape, which limits the detection sensitivity. ${ }^{25,26}$ A schematic of a typical QEPAS setup, as used in most reported QEPAS sensor systems, is shown in Fig. 1. A laser source, typically a quantum cascade laser (QCL) or an interband cascade laser (ICL), serves as the excitation source 


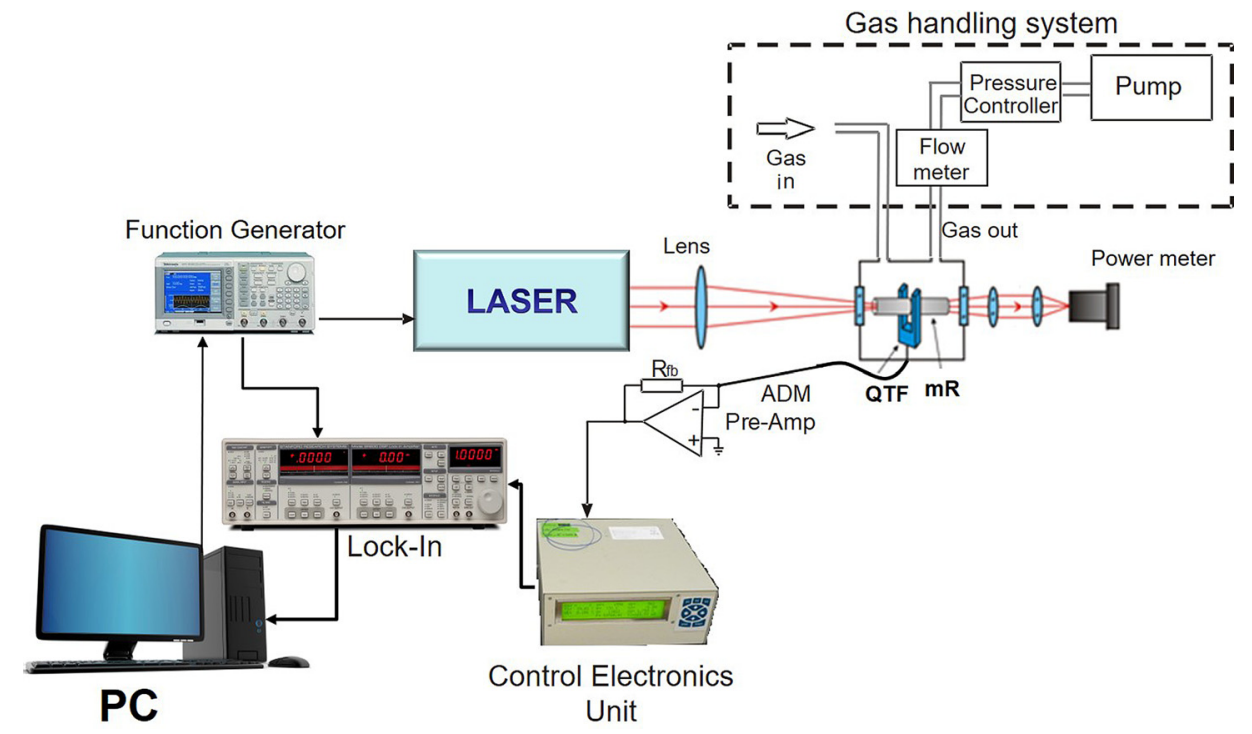

FIG. 1. Schematic of a typical QEPAS sensor setup. QTF, quartz tuning fork; $\mathrm{mR}$, acoustic micro-resonator; ADM, acoustic detection module; PC, personal computer.

for generating the QEPAS signal. The ADM is mounted inside a vacuum-tight cell equipped with optical windows. The laser beam is focused by a lens between the QTF prongs and it passes through the ADM. A power meter is used for monitoring the laser power as well as for optical alignment. A function generator is used to sinusoidally either the laser current at the QTF resonance frequency or one of its subharmonics.

The flow rate and pressure of the sample gas passing through the ADM can be controlled and maintained by using a flow meter and pressure controller, respectively. A control electronics unit (CEU) is used to determine the main QTF parameters: dynamic resistance $R$, quality factor $Q$, and resonant frequency $f$. The CEU is also designed to pass on the amplified signal from a transimpedance pre-amplifier (PreAmp) to the lock-in amplifier to demodulate the QEPAS signal. A picture of a recently realized ADM is shown in Fig. 2.

QEPAS based sensor systems are usually operated with a wavelength modulation (WM) and $2 f$ detection configuration in order to suppress background noise originating from spectrally nonselective absorbers (such as the mR, QTF, and the optical windows of the ADM). One important advantage of WM is that only the noise centered within the detection bandwidth will affect trace-gas measurements. In WM, the laser beam is wavelength modulated at $f / 2$ frequency and the lock-in amplifier demodulates the QTF response at $f$.

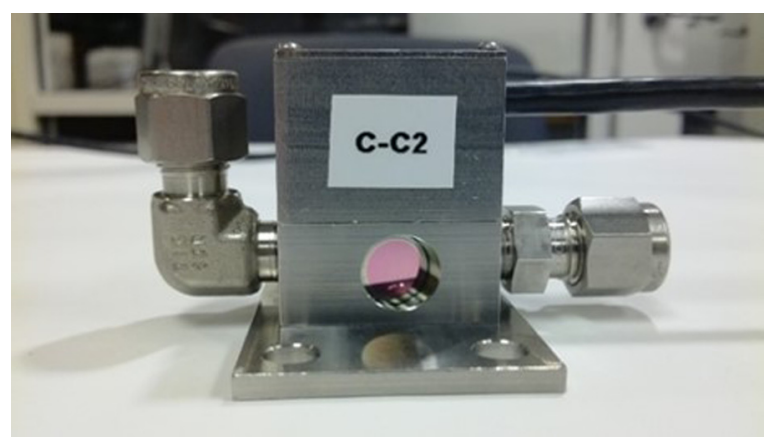

FIG. 2. Photograph of an ADM. An optical window and the gas inlet and outlet connectors are also visible.
Spectral data can be acquired when the laser wavelength is scanned. For broadband gas species with unresolved rotational spectral structure, the WM approach is not usable since the laser source cannot be wavelength modulated with a sufficient spectral tuning coverage. Thus, the only possibility is to operate in an amplitude modulation (AM) configuration, where the laser is modulated at $f$ and the QEPAS signals are detected by the lock-in amplifier at the same $f$ frequency. Unlike WM QEPAS, AM QEPAS is not background free. However, this AM QEPAS background can be stable over several hours and hence allows background subtraction. ${ }^{27-29}$

\section{B. Fiber-based QEPAS}

One of the critical issues of a QEPAS sensing system is the optical alignment. Different approaches, starting from a standard solution based on focusing lenses, have been implemented to focus the laser light between the QTF prongs and through the $\mathrm{mR}$, with particular care in avoiding that the laser beam illuminates the ADM. This condition is more easily fulfilled if the laser beam power distribution resembles a Gaussian $\mathrm{TEM}_{00}$ profile, as otherwise the use of a pinhole to filter out beam tails becomes necessary. Spatial filters reduce the optical power focused through the QTF and consequently reduce the QEPAS signal. An effective solution for improving the mid-IR laser beam profile is the use of single-mode optical fibers. Both hollow- ${ }^{23-33}$ and solid-core ${ }^{34-36}$ fibers have been demonstrated to be very efficient for both near-IR diode lasers as well as mid-IR ICL and QCL beam spatial cleaning and can cover the $2 \mu \mathrm{m}-12 \mu \mathrm{m}$ spectral range. A schematic of the optical coupling between laser excitation source and the ADM via an optical fiber is shown in Fig. 3(a). A laser output focuser is mounted at the fiber exit in order to provide a focusing distance of several centimeters and produce laser beam shaping of the light exiting from the fiber and to be transmitted through the ADM without hitting it. Several QEPAS sensors exploiting single mode mid-IR fibers providing enhanced versatility in terms of flexible laser beam guidance and compactness have been demonstrated. ${ }^{16,24,37-40}$ Another way to guide the laser light through the QTF and generate pressure 
a)
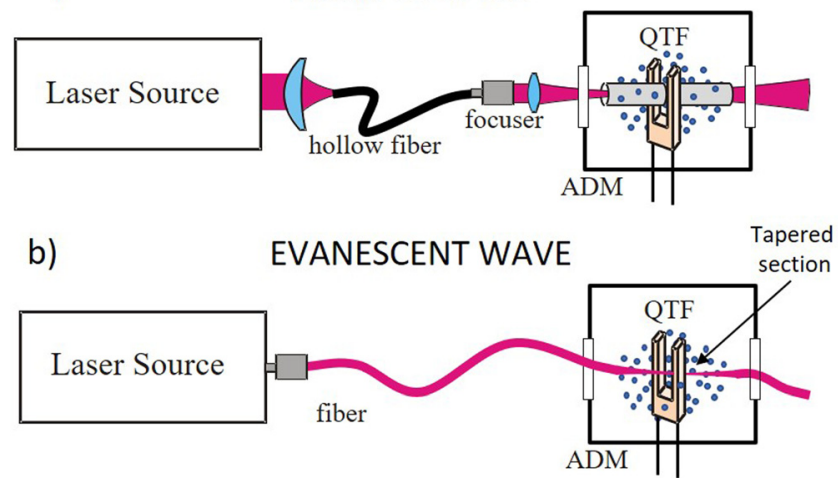

FIG. 3. (a) Schematic of the single-mode fiber-coupling between the laser source and the ADM and (b) evanescent-wave approach to excite a gas sample (blue dots).

waves between the prongs is the exploitation of the evanescent wave effect in optical fibers. ${ }^{41-44}$ This approach has been demonstrated in the near-IR and is based on fiber tapering. A portion of the tapered fiber is positioned in the gap between the two prongs of the QTF. The laser radiation is guided along the fiber with a very small beam size and the light coming out from the tapered portion of the fiber interacts with the gas sample via the evanescent wave effect. A schematic of the evanescent-wave approach to excite the gas sample is shown in Fig. 3(b).

The evanescent field generated in the tapered section positioned between the QTF prongs can be absorbed by the target gas molecules surrounding the fiber, thereby generating acoustic pressure waves that are detected by the QTF.

\section{Modulation cancellation method (MOCAM)}

Another effective method for reducing or cancelling the background noise generated by portion of the laser beam hitting the ADM can be accomplished by the modulation cancellation method (MOCAM). MOCAM is a variation of modulation spectroscopy using two light sources. ${ }^{45-47}$ The basic concept of MOCAM is that the powers and modulation phases of two light sources can be adjusted to balance out the background signal, especially that caused by the stray light, which limits the measurement accuracy. MOCAM can detect a small signal in the presence of a large background noise by physical cancellation of the sensor response. Different approaches have been demonstrated for MOCAM. In a configuration exploiting to demonstrate trace gas detection of broadband absorber species by use of wide stripe diode lasers ${ }^{45}$ a balance laser source, whose emission wavelength is located outside the target gas absorption band, is used to intentionally generate an identical background noise to that caused by exciting laser source, with an emission wavelength falling within the absorption band of the target gas. A schematic of this approach is shown in Fig. 4.

If the two lasers are modulated in counter-phase, the QTF prongs are forced to vibrate in opposite direction by the two laser sources and, as a result, no QTF vibrations are produced, and thereby not generating a background noise signal. Other applications for MOCAM include measurements of

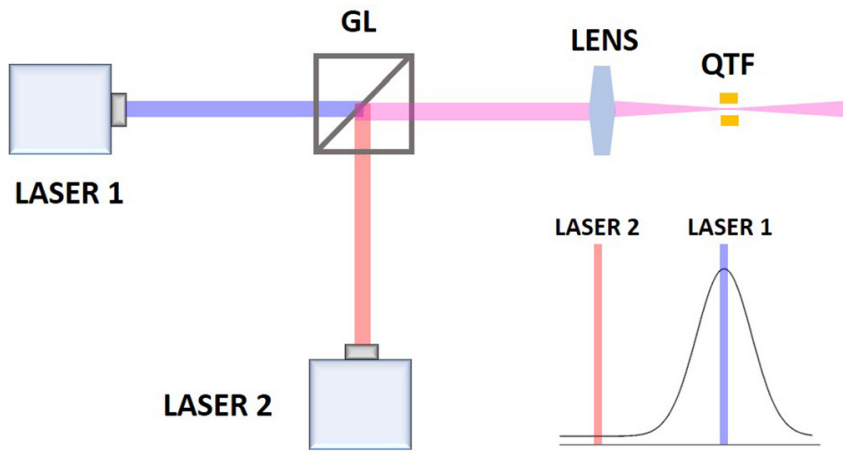

FIG. 4. Schematic of the MOCAM approach for detection of broadband absorbers; GL: Glann Prism.

small temperature difference in a gas mixture ${ }^{46}$ and analysis of isotopic composition. ${ }^{47}$ For these types of measurements, the output powers and modulation phases of the two lasers resonant with two selected absorption lines are adjusted in such a way that the signal detected from the reference sample is zero. Then, in an appropriately designed optical configuration, the signal from the analyzed sample will be proportional to the deviation of the absorption line-strength ratio from the reference sample line-strength ratio. A variant of MOCAM was the implementation of an electrical driving signal to induce opposite QTF vibrations with respect to those generated by the exciting laser source. This approach is called electronic MOCAM (E-MOCAM). The E-MOCAM method makes it possible to use commercial high-power light-emitting diodes (LEDs) ${ }^{48}$ or fiber-amplified near-IR sources, ${ }^{49,50}$ characterized by limited beam qualities, as excitation sources in on-beam QEPAS sensors, by suppressing the background noise caused by stray light by up to three orders of magnitude.

\section{Beat frequency (BF) quartz-enhanced photoacoustic spectroscopy (BF-QEPAS)}

A QTF flexural resonance mode with a high $Q$-factor is advantageous in terms of QEPAS detection sensitivity [see Eq. (1)]. Indeed, the detected photoacoustic signal is proportional to the QTF resonance $Q$-factor or more precisely to the effective QTF integration time $t=Q / \pi f_{0},{ }^{51}$ if we consider a fast energy relaxing target analyte. At low pressures, values over $1 \mathrm{~s}$ can be reached for bare QTFs, ${ }^{52}$ reducing to a few hundreds of ms when implementing a mR systems. ${ }^{2}$ In order to allow the acoustic mode of the resonator to accumulate all the absorbed laser energy, the measurement period should be 3 to 4 times longer than $t$, which means a few hundreds of $\mathrm{ms}$ for uncorrelated trace-gas concentration measurements. This time interval is acceptable for applications, such as environmental monitoring where no fast changes in the gas sample composition occur. However, this time interval is too long for rapid scan trace-gas measurements required for applications like multi-line detection of the same or different gas species, where a scan rate $>1 \mathrm{~Hz}$ is required. ${ }^{53} \mathrm{~A}$ recent and innovative QEPAS technique approach relying on beat frequency (BF) signals between the QTF resonance frequency $f$ and the laser modulation frequency was reported in Ref. 51. The BF-QEPAS concept requires that the laser 
modulation frequency differs by $\Delta f$ with respect to the QTF resonance frequency $f$. For this condition, a BF-QEPAS signal with a period of $\Delta t$ is generated when the laser wavelength is rapidly scanned across a targeted absorption line and the QTF signal is demodulated at the same frequency $f$.

In BF-QEPAS, an acoustic pulse induced by the target gas absorption is generated as a result of rapid wavelength scanning $\left(>30 \mathrm{~cm}^{-1} \mathrm{~s}^{-1}\right)$, which causes the prongs of the QTF to vibrate in a short period of time. Subsequently, while the QTF relaxes the vibrational energy at its natural mode frequency via loss mechanisms, the QTF signal is demodulated at the laser modulation frequency $f$. When the averaging time is short enough $(<100 \mathrm{~ms})$ to provide a sufficient system detection bandwidth, a BF signal with an exponential decay envelope is generated from the QTF-transient response. The concentration of the trace gas, the resonance frequency, and $Q$-factor of the QTF can be obtained by measuring the peak amplitude, $\Delta t$ and the decay time $\tau$ of the BF-QEPAS signal, respectively. An example of the acquired signal is shown in Fig. 5.

Using this approach, it is possible to retrieve the QTF resonance frequency $f$ and its $Q$-factor, as well as, the target gas concentration, all in measurement times as short as $30 \mathrm{~ms}$. Such a method provides a comparable detection sensitivity with respect to conventional QEPAS when detecting fast relaxing molecules such as $\mathrm{H}_{2} \mathrm{O}$. It is worth noting that the BF-QEPAS technique can be employed in any QEPASbased sensor systems without any hardware changes of a conventional QEPAS ADM system.

\section{E. Intracavity QEPAS}

The QEPAS technique is characterized by direct proportionality between the signal amplitude and the laser power available for gas excitation. An increase of the optical power focused between the QTF prongs improves the minimum detection limit of a QEPAS sensor. Approaches like multipass and/or cavity optical build up are used to increase the interaction length of the laser beam in the gas sample. The first demonstration of a QEPAS sensor exploiting a build-up

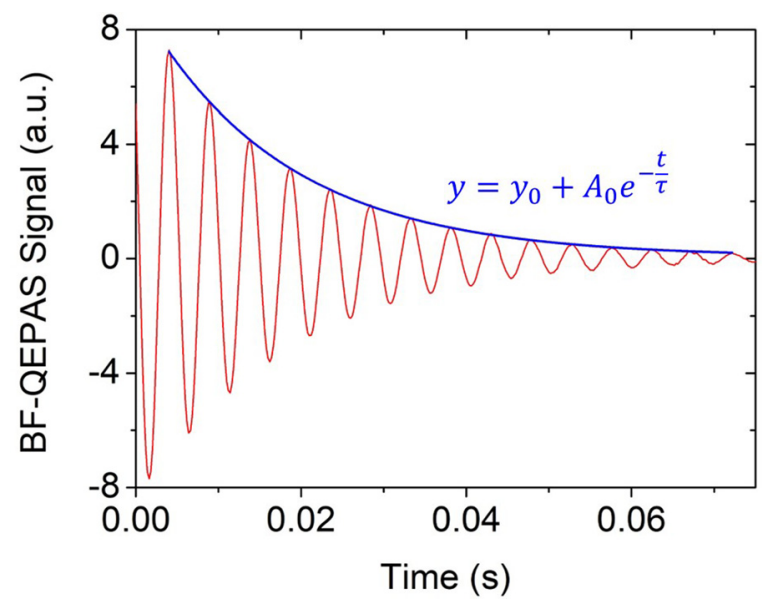

FIG. 5. Experimental results of BF-QEPAS obtained with $2.5 \%$ water vapor at room temperature and atmosphere pressure. The blue exponential line represents the best fit of the local maxima of the BF-QEPAS signal, which allows to extract a decay time $\tau=17.5 \mathrm{~ms}$. The period of the oscillation is $\Delta t=4.9 \mathrm{~ms}$. cavity with high-finesse for laser optical power enhancement was reported in Refs. 54 and 55. This novel QEPAS approach combines QEPAS with cavity enhanced absorption spectroscopy (CEAS) and is called intra-cavity QEPAS (IQEPAS). Using this configuration, $\mathrm{CO}_{2}$ detection sensitivities in ppt concentration range were achieved. ${ }^{54} \mathrm{~A}$ schematic of the I-QEPAS configuration is shown in Fig. 6.

In the first I-QEPAS demonstration, a bow-tie resonator system configuration composed by two concave mirrors and two plane mirrors was implemented. The quartz tuning fork (QTF) was positioned at the cavity waist between the two concave mirrors. In I-QEPAS experiments, it is crucial to lock the resonance frequency of the cavity to the laser during the slow linear scan time in order to retrieve a spectral scan of a selected gas absorption line. The simplest way is to adjust the cavity length by using a piezoelectric actuator attached to one of the cavity mirror mounts. The improvement in terms of sensitivity with respect to conventional QEPAS setup (operating under the same conditions of molecular linewidth, pressure and laser output power) is equal to the power enhancement factor occurring in the optical resonator ( 240 in Refs. 54 and 55) Recently, novel compact bow-tie cavities capable to reach power enhancement factors over 300 have been realized, ${ }^{56,57}$ promising even further improvements in detection sensitivity. Since to-date a bare QTF was used in I-QEPAS based sensors. Additional improvements are expected by adding a $\mathrm{mR}$ to the photoacoustic detection module, potentially leading to the realization of sensors with a ppq detection limit. However, a useful figure of merit to compare different types of QEPAS sensors is the normalized noise equivalent absorption (NNEA). The minimum detectable absorption coefficient $\left(\alpha_{\min }\right)$ can be normalized with the laser power $P_{L}$ and the detection bandwidth $\Delta f_{B}$ to determine NNEA, defined as NNEA $=\mathrm{P}_{\mathrm{L}} \cdot\left(\alpha_{\min }\right) / \sqrt{ } \Delta \mathrm{f}_{\mathrm{B}}$. Typical NNEA values reached with mid-IR QEPAS sensors fall in the range of $10^{-8}-10^{-9} \mathrm{~W} \cdot \mathrm{cm}^{-1} \mathrm{~Hz}^{-1 / 2}$ with an integration time of $1 \mathrm{~s}$ and assuming typical optical powers of a few tens of milliwatt. ${ }^{2}$ Taking advantage from the optical power build-up occurring in the cavity, I-QEPAS experiences a reduction of the $\alpha_{\min }$ with respect to the QEPAS technique when the same laser source is used (which means the same $\mathrm{P}_{\mathrm{L}}$ value), corresponding to a reduction of the NNEA value to the $10^{-12} \mathrm{~W} \mathrm{~cm}^{-1} \mathrm{~Hz}^{-1 / 2}$ range.

\section{F. QEPAS configuration based on dual micro-resonators and dual quartz tuning forks (QTFs)}

A simplified model, which considers the total momentum of a pressure force acting on the two prongs of the QTF

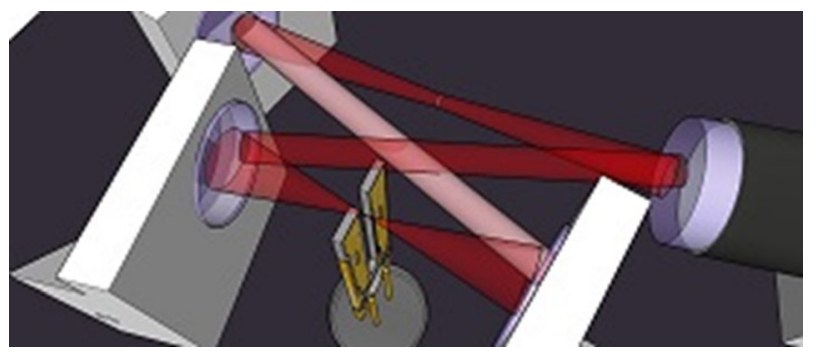

FIG. 6. Schematic of the I-QEPAS configuration. 
due to a point-source sound wave positioned between the QTF prongs, has allowed calculating the optimal laser beam focus position with respect to the vertical axis for a bare QTF. $^{2}$ The results agree with a more detailed theoretical model of a QEPAS sensor proposed by Petra et al. ${ }^{58}$ and show a very good agreement with experimental results. ${ }^{2}$ For example, for a standard QTF operating at $32 \mathrm{kHz}$, the optimal vertical position is located at $0.33 \mathrm{~mm}$ below the QTF opening and centered between its tines. ${ }^{2}$ However, the presence of a $\mathrm{mR}$ and its interaction with the QTF alter the acoustic characteristics of the ADM, and the optimal focus position has to be experimental determined. An experimental investigation of the $\mathrm{mR}$ position effects was reported in Refs. 59 and 60 and demonstrated that the QEPAS signal-tonoise plot as a function of the $\mathrm{mR}$ vertical position shows a maximum centered on a nearly flat curve. This flat curve region is sufficiently wide that it allows the positioning of two mRs in a QTF, as shown in Fig. 7. This novel QEPAS configuration has been referred to as "double mR QEPAS."

The lowering of the ADM $Q$-factor due to the acoustic coupling of the QTF with the two mRs leads to an ADM response time 20 times faster compared with a bare QTF and to a wider response bandwidth. Hence, the signal amplitude and phase of the double $\mathrm{mR}$ spectrophone become insensitive to small drifts of the resonant frequency compared to the high- $Q$ bare QTF and to the single $\mathrm{mR}$ spectrophone. Such a double $\mathrm{mR}$ configuration can provide two advantages: (1) two laser excitation sources from different optical wavelength scans perform the optical signal addition or cancellation by means of the spectrophone, avoiding laser beam combination as required by MOCAM and (2) the spectrophone can implement ultrafast two-gas measurements by switching between two detection channels. Performing a two spectra signal cancellation with the line-locked mode of two lasers at a specific concentration ratio of two target gases, the double $\mathrm{mR}$ spectrophone is able to measure directly the deviation of the concentration ratio. The deviation can then be used as a feedback to control gas valves to maintain a specific gas mixing ratio, which is important for controlling gas chemical reactions, gas mixtures or optimizing combustion processes.

Another configuration relies on the use of two QTFs in a single ADM. ${ }^{61}$ In this multi-quartz-enhanced photoacoustic

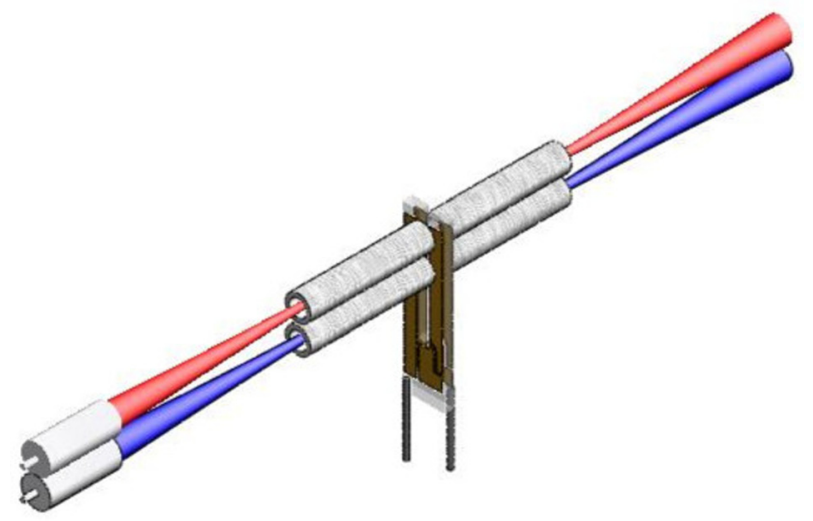

FIG. 7. Schematic of double mRs QEPAS spectroscopy (M-QEPAS) sensor system, the acoustic wave signal is detected by multiple QTFs simultaneously and the electrical signals generated by the QTFs is added in order to increase the effective signal amplitude. A schematic of the M-QEPAS configuration is shown in Fig. 8.

The results reported in Ref. 60, showed that, compared with QEPAS sensor using a bare single QTF, M-QEPAS employing two bare QTFs provided a signal enhancement of 1.7 times for the same operating conditions. Further improvement in detection sensitivity of the M-QEPAS configuration was achieved by employing acoustic micro-resonator tubes for both QTFs, as demonstrated in Ref. 62. Two kinds of multi-QTF based “on-beam" QEPAS spectrophones were realized. Schematics of these two configurations are shown in Fig. 9.

When two QTFs were placed close to each other and in parallel and coupled with two mRs [see Fig. 9(a)], the $Q$-factor of the multi-QTFs system becomes higher than that of each QTF in a "standard" on-beam configuration using the same $\mathrm{mR}$ system. This configuration yielded a 1.4 times enhancement compared to the traditional single QTF based "on-beam" spectrophone and a 33 times enhancement compared to the single bare QTF based spectrophone. For the configuration shown in Fig. 9(b) with the two QTFs spaced by one of the $\mathrm{mR}$ tubes, the $Q$-factor increases with respect to the two QTFs in a "standard" on-beam configuration, but the QEPAS signal is 1.6 times higher than that of the traditional single QTF based “on-beam" spectrophones signal and 36 times higher than that of the single bare QTF based spectrophone. The signal enhancement can be further improved by using a phase shifter to adjust the phase difference among

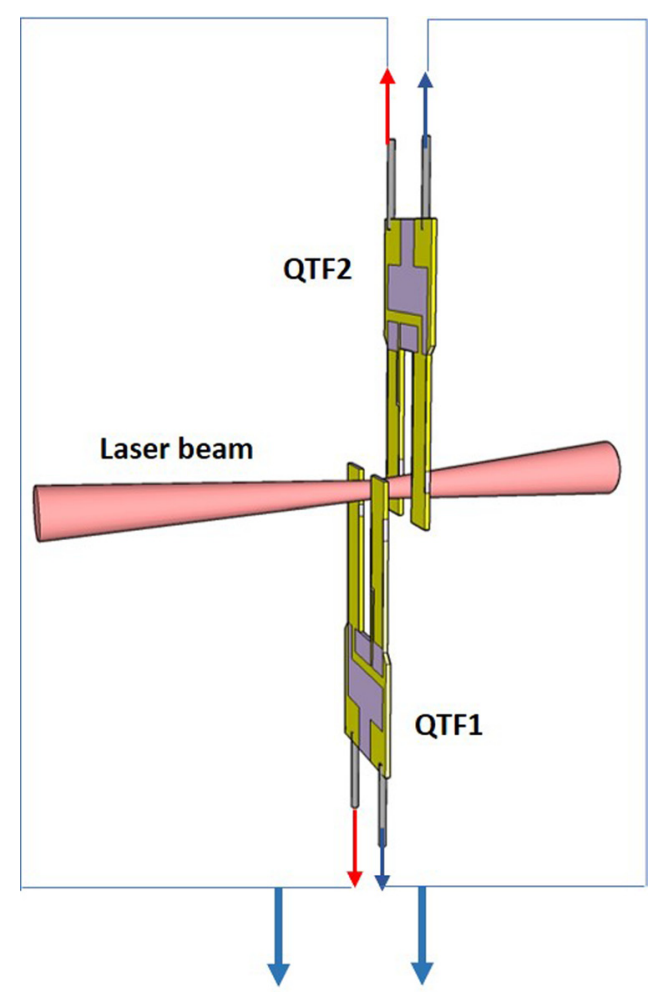

FIG. 8. Schematic of M-QEPAS with two QTFs. 


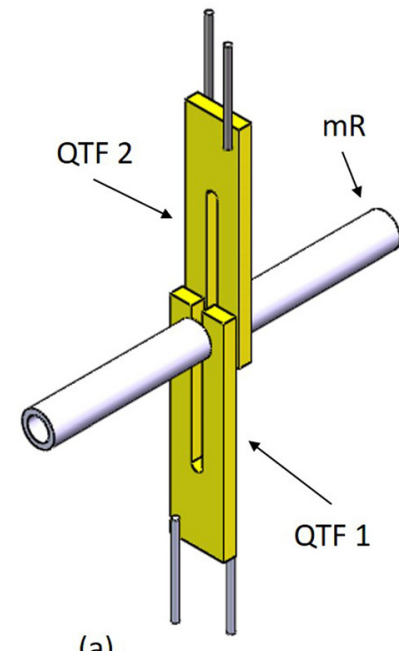

(a)

(b)

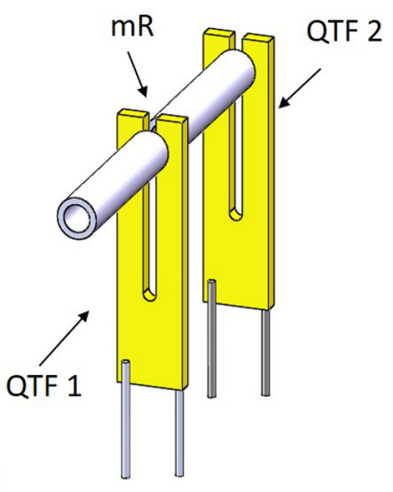

FIG. 9. (a) and (b) Two kinds of multi-QTF based "on-beam" spectrophones.

multi-QTFs and a capacitance in series with one of the QTFs to perfectly match the resonance frequencies.

\section{QEPAS WITH CUSTOM QTFS}

Prior to 2013, all the QEPAS sensors reported in the literature employed commercial standard QTFs operating at the fundamental in-plane flexural resonant mode, with a frequency of $\sim 32.7 \mathrm{kHz}$. However, the standard QTFs structure and its operating frequency were optimized for timing purposes and not for spectroscopic applications. Two main factors pose limitations to the use of standard QTFs for QEPAS sensing. First, the QEPAS signal depends strongly on the energy relaxation rates associated with the targeted gas species. The relaxation time constant $\tau_{\mathrm{T}}$ depends on the specific molecule and on the specific gas carrier (typically either air or $\mathrm{N}_{2}$ ) and intermolecular interactions. For gases, vibrational-translational $(\mathrm{V}-\mathrm{T})$ time constants $\tau_{\mathrm{T}}$ fall typically in the $\mu$ s temporal range. ${ }^{63}$ To ensure that the energy transfer occurs efficiently for a fast modulation $f$ of the incident laser radiation, it is necessary to satisfy the condition $f \ll 1 / 2 \pi \tau_{T}{ }^{64}$ Therefore, QEPAS is more sensitive to the energy relaxation rate compared to the conventional PAS, which is commonly performed at $f<4 \mathrm{kHz} .{ }^{10,65}$ For gas species with a slow relaxation rate (such as $\mathrm{CO}, \mathrm{CO}_{2}$ and $\mathrm{NO}$ ), the thermal waves in the gas cannot follow fast changes of the laser induced molecular vibration excitation. Thus, the generated photoacoustic wave is weaker than it would be in case of fast V-T energy equilibration. ${ }^{2}$ Second, standard $32 \mathrm{kHz}-\mathrm{QTF}$ are characterized by a small volume between their prongs $\left(\sim 0.3 \times 0.3 \times 3 \mathrm{~mm}^{3}\right)$. Hence, the use of light sources with a limited beam quality, like LED, a fiber amplified laser, or a laser emitting in the $\mathrm{THz}$ spectral region $(\lambda>30 \mu \mathrm{m})$, is precluded, since they do not allow adequate focalization of the excitation beam between the prongs spaced by $\sim 300 \mu \mathrm{m}$. The best method to circumvent these limitations is to employ QTFs with larger prongs spacing and operation frequencies possibly $<20 \mathrm{kHz}$ to approach gas relaxation rates, but typically a decrease in $f$ leads to a decrease of the resonance $Q$-factor, ${ }^{66}$ so that a trade-off optimization of the above parameters must be found. In Secs. III A-III H, we will review the results obtained in modelling, design, and development of novel QTFs devoted to QEPAS gas sensing systems.

\section{A. QTF modeling}

A QTF can be considered as two identical cantilevers coupled by a low-loss quartz bridge. A schematic of a QTF is shown in Fig. 10.

The in-plane flexural vibrations modes of the two prongs can be classified as in phase (symmetric) and out of phase (antisymmetric). The flexural antisymmetric modes, in which the two prongs oscillate in antiphase, are the only vibrational modes employed in QEPAS based sensor systems. ${ }^{67}$ The resonance frequencies of the QTF can be calculated in the approximation of an independent cantilever vibrating in inplane modes. Each prong of the tuning fork can be treated as a clamped beam. In this approximation, the frequencies of the vibrational modes are obtained by including a free-motion condition on one boundary of the prong and a clamped condition on the other (clamped-free boundary condition) and then solving for the propagation of a shear acoustic wave. Assuming that an elastic Young modulus $E$ of the QTF material, a moment of inertia $I$ of the prong, and a cross-sectional area $A=T \cdot W$ are constant along the prong length for small amplitude oscillations, the motion of each prong can be described using a one-dimensional model and the resonance frequencies in vacuum are given by ${ }^{68}$

$$
f_{n, v a c}=\frac{\pi T}{8 \sqrt{12} L_{p}^{2}} \sqrt{\frac{E}{\rho}} \nu_{n}^{2},
$$

where $\rho=2650 \mathrm{~kg} / \mathrm{m}^{3}$ is the quartz density, $E=72 \mathrm{GPa}$ is the component of the quartz Young's modulus in the vibrating plane of the QTF. $L_{p}$ and $T$ are the tuning prong length and thickness, as depicted in Fig. 10. The first two eigenmodes values are obtained for $\nu_{n=0}=1.194$ (fundamental mode) and for $\nu_{n=1}=2.988$, (first overtone mode). Let us consider a QTF vibrating in a fluid medium. The vibration will induce a motion of the fluid and thus the QFT will

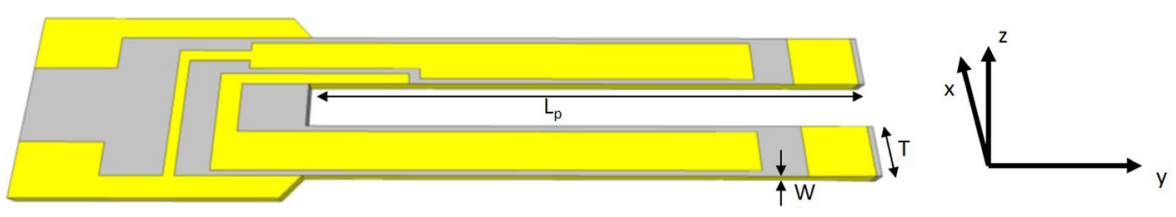

FIG. 10. Schematic of a custom QTF with a gold contact pattern. The coordinate system with the y-axis parallel to the beam axis of the prong is depicted. In this way, the prongs bending for in-plane flexural modes occur in the xy-plane. 
experience an energy loss (part of the energy is transferred to the fluid or to the bulk) and additional inertia. Therefore, this situation can be modelled by adding a reaction force of the fluid consisting of two terms: a resistive term (which leads to energy dissipation by acoustic loss) and a reactive term (which leads to the additional inertia of the QTF). Taking into account both effects, the Euler-Bernoulli equation expresses the vibrational profile of the single prong results ${ }^{69}$

$$
E I_{y} \frac{\partial^{4} y}{\partial^{4} x}+C_{d} \frac{\partial y}{\partial t}+(\rho A+u) \frac{\partial^{2} y}{\partial t^{2}}=0
$$

where $C_{d}$ is the damping parameter, which accounts for energy loss and $u$ is the added mass per unit length, $t$ is the time, and $x$ and $y$ directions are represented in Fig. 10. If the damping parameter is negligible, the extra mass term causes a variation of the resonant frequencies of the QTF flexural modes, which now can be calculated as

$$
f_{n}^{\prime}=\frac{\pi}{8 L^{2}} \sqrt{\frac{E I}{(\rho A+u)}} \nu_{n}^{2} .
$$

If the relation $u \ll \rho \cdot A$ is verified, then is possible to calculate the frequency shift $\Delta f$ due to gas as

$$
\Delta f=\frac{f_{n}-f_{n}^{\prime}}{f_{n}^{\prime}}=-\frac{1}{2} \frac{u}{\rho A} .
$$

The added mass is proportional to the density $\rho_{0}$ of the fluid medium. Since the relationship between the pressure $P$ and the density $\rho_{0}$ in a gas is $P \propto \rho_{0}, \Delta f$ varies linearly with the gas pressure and it is possible to rewrite Eq. (5) as

$$
f_{n}(p)=f_{n, v a c}-k_{p, n} P
$$

where $k_{p, n}=f_{n, v a c} \cdot u /(2 \rho \cdot W \cdot T)$. The energy dissipation due to acoustic losses in the fluid leads to a reduction of the $Q$-factor of the flexural mode resonance. A fluid damping parameter can be introduced, which is proportional to the density $\rho_{o}$ and the viscosity $\eta$ of the fluid. Assuming that $P \propto \rho_{o}$ and $\eta$ does not noticeably change with $P$, the influence of the fluid damping on $Q$ can be expressed in terms of the energy loss $1 / Q(P)$ at the gas pressure, and considering the damping parameter in the form $C_{d}=a \sqrt{ } P,{ }^{70}$ where $a$ is a parameter specific of the tuning fork, the dependence of the $Q$-factor from the pressure results in

$$
Q(P)=\frac{Q_{0}}{1+Q_{0} a \sqrt{P}},
$$

where $Q_{0}$ is the $Q$-factor under vacuum conditions. This analysis describes the behavior of a cantilever beam. Therefore, it is feasible to use a single quartz cantilever beam of the size of the fork's prong as a photoacoustic transducer. In this case, a tuning fork is a system of two coupled cantilevers oscillating in opposition. The result is that, in spite of its arms' motion, the center of mass remains fixed in position. In contrast, a resonating single cantilever beam has an oscillating center of mass. This motion dissipates energy, while this energy dissipation channel is not present in a wellbalanced QTF. In a cantilever, the losses due to the center of mass motion are generally large and lead to a reduction of the $Q$ by several orders of magnitude. This explains why only QTFs are used for QEPAS applications.

\section{B. Custom QTFs design and characterization}

While the resonance frequencies $f_{n, v a c}$ can be estimated using Eq. (3), a prediction of the QTF $Q$-factor via an analytical method is not possible, since too many extrinsic and intrinsic losses mechanisms contribute to its value. Extrinsic losses mechanisms are due to interactions with the surrounding medium, while the intrinsic losses mechanisms include support losses (interactions with its support structure) as well as thermo-elastic losses. In addition, less predictable factors such as processing anisotropy, crystal quality, and gold patterning contribute to the final $Q$-factor value. In Refs. 67 and 71, a set of QTFs with different values of prong spacing, lengths, and thicknesses as well as crystal thickness were designed, fabricated, and evaluated, leading to a determination of the dependence of the QTF parameters and performance on their relevant dimensions. The investigated QTF samples' dimensions are listed in Table I, while Fig. 11 shows the schematic of seven different QTF designs.

The QTFs were realized starting from a z-cut quartz wafer with a $2^{\circ}$ rotation along the $\mathrm{x}$-axis. The $\mathrm{z}$-cut is the dominant low frequency (up to $50 \mathrm{kHz}$ ) crystal-cut, which provides thermally stable flexural vibrational modes frequencies. Standard photolithographic techniques were used to etch the QTFs. Cr and Au patterns are deposited on both sides of the wafer. The main QTF parameters (resonance frequencies, related $Q$-factors, and electrical resistance) were determined by electrically exciting the QTF with a sinusoidal voltage signal generated by an arbitrary function generator (AFG). The current signal is collected and converted into a voltage signal by a transimpedance amplifier and sent to a lock-in amplifier, which demodulates the signal at the same

\begin{tabular}{|c|c|c|c|c|c|c|c|}
\hline Parameters & QTF standard & QTF \#1 & QTF \#2 & QTF \#3 & QTF \#4 & QTF \#5 & QTF \#6 \\
\hline$L_{p}(\mathrm{~mm})$ & 3.0 & 3.5 & 10.0 & 10.0 & 11.0 & 17.0 & 16.8 \\
\hline$W(\mathrm{~mm})$ & 0.34 & 0.25 & 0.25 & 0.5 & 0.25 & 0.25 & 0.8 \\
\hline$T(\mathrm{~mm})$ & 0.35 & 0.2 & 0.9 & 1.0 & 0.5 & 1.0 & 1.4 \\
\hline Prong spacing $(\mathrm{mm})$ & 0.3 & 0.4 & 0.8 & 0.5 & 0.6 & 0.7 & 1.0 \\
\hline
\end{tabular}
angular frequency set by the AFG. A schematic of the setup used for these measurements is shown in Fig. 12.

TABLE I. Dimensions of standard and custom tuning forks studied in Refs. 67 and 71: $L_{p}$ (QTF prong length), $T$ (thickness of the prong), $W$ (thickness of the quartz crystal). 


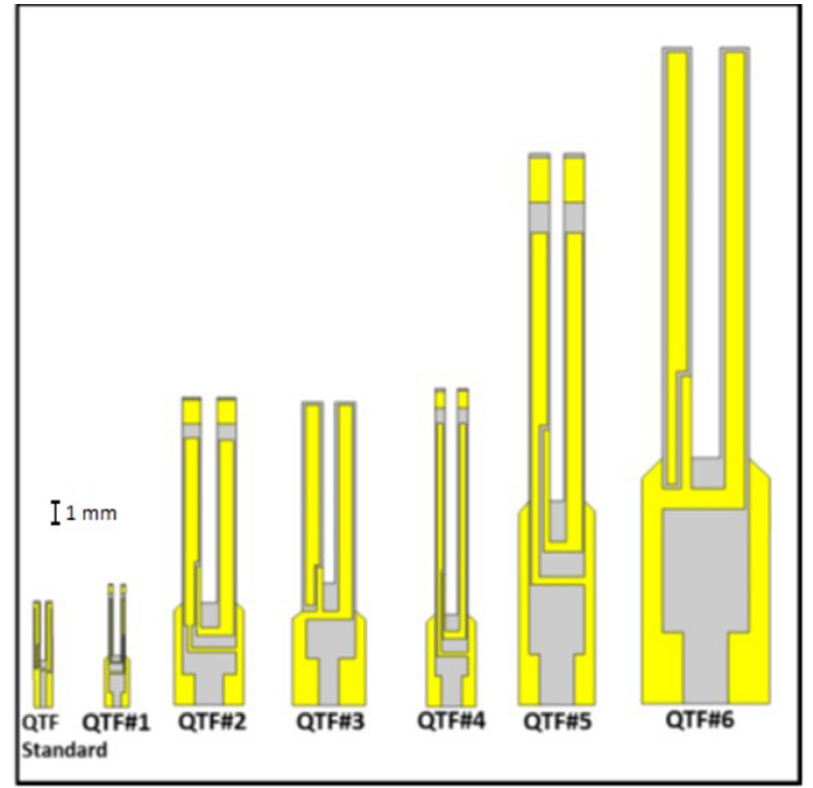

FIG. 11. $\mathrm{X}-\mathrm{z}$ plane view of standard and custom designed tuning forks. The size scale is indicated on the left side. The grey areas represent uncovered quartz, while the gold-yellow area represents gold electrode patterns.

The extracted resonance frequency of the fundamental $f_{O(\exp )}$ flexural mode and the related $Q_{0}$-factors under vacuum conditions are compared in Table II, together with the theoretical $f_{O, v a c}$, calculated using Eq. (4). In Table II, the electrical resistances $R$ and the $Q$-factor $\left(Q_{a t m}\right)$ measured at atmospheric pressure are also listed.

The small discrepancies $(<5 \%)$ between experimental and theoretical values are due to additional weight of the electrode gold layers, dependence of the elasticity modulus of quartz on the crystallographic axes orientation, and deviations in geometry between the modelled and the actual QTFs.

In Fig. 13, the results obtained by plotting the QTFs resistances and $Q$-factors measured for the QTFs fundamental vibrational mode at atmospheric pressure (see Table II) versus the geometrical parameters of the QTFs are shown. Theoretically, $R$ is related to geometrical parameters, since $R \sim L_{p}{ }^{2} / W \cdot T^{1 / 2}{ }^{68}$ A linear correlation is observed between resistances and $L_{p}{ }^{2} / W \cdot T^{1 / 2}$ values, except for QTF\#1 and QTF\#4 where the discrepancy is due to the different charge
TABLE II. Experimental and calculated physical parameters for the QTFs: resonance frequencies of the fundamental mode $f_{O \text {,vac }}$ (calculated) and $f_{O}{ }^{(\exp )}$ (measured) and related quality factor $Q_{0}$. The electrical resistances $R$ and the $Q$-factor $\left(Q_{\text {Atm }}\right)$ measured at atmospheric pressure are also listed.

\begin{tabular}{lccccc}
\hline \hline QTF type & $\mathrm{f}_{0, \mathrm{vac}}(\mathrm{Hz})$ & $\mathrm{f}_{0(\exp )}(\mathrm{Hz})$ & $\mathrm{Q}_{0}$ & $\mathrm{Q}_{\text {Atm }}$ & $\mathrm{R}(\mathrm{k} \Omega)$ \\
\hline QTF standard & 32743.61 & 32762.84 & 88719 & 13690 & 94.6 \\
QTF \#1 & 13746.59 & 14049.20 & 10862 & 3980 & 1913.3 \\
QTF \#2 & 7577.81 & 7230.27 & 36564 & 8400 & 301.7 \\
QTF \#3 & 8419.79 & 8439.51 & 47020 & 13150 & 455.6 \\
QTF \#4 & 3479.25 & 3456.69 & 23282 & 3880 & 990.2 \\
QTF \#5 & 2913.42 & 2869.07 & 34800 & 5210 & 810.1 \\
QTF \#6 & 4176.48 & 4250.01 & 50130 & 17850 & 194.1 \\
\hline \hline
\end{tabular}

collection efficiency determined by the gold contacts QTF patterns. Noteworthy is the linearity between $Q$-factors versus $W T / L_{p}$ values [see Fig. 13(b)]. This result allows to predict empirically the trend of the $Q$-factor from the prongs size.

For QEPAS applications, $R$ should be kept low and the $Q$ as high as possible. Both conditions can be obtained by increasing $W$ and the ratio $T / L_{p}$. However, the resonance frequency $f$ increases with $T / L_{p}{ }^{2}$ and $f$ should not exceed $40 \mathrm{kHz}$ since the thermal waves in the gas cannot follow rapid changes of the laser induced molecular vibration excitation. Hence, custom QTFs have been used for QEPAS sensing applications and led to new approaches in QEPAS based sensor technology as reported in the following paragraphs.

\section{QEPAS in the THz range}

The $\mathrm{THz}$ range is the most promising spectral range for QEPAS applications provided that efficient non-cryogenic, compact $\mathrm{THz}$ laser sources will be realized in the near future. In the $\mathrm{THz}$ spectral range, gas absorption is mostly due rotational-translation (R-T) molecular transitions and the related relaxation rates are up to three orders of magnitude faster with respect to V-T transitions in the mid-IR. ${ }^{63}$ If operating with fast relaxing transitions, it is possible to operate a QEPAS based gas sensor at low pressures. With this condition, the energy losses due to mechanical viscosity and friction from the gas are reduced and the $Q$-factor increases,

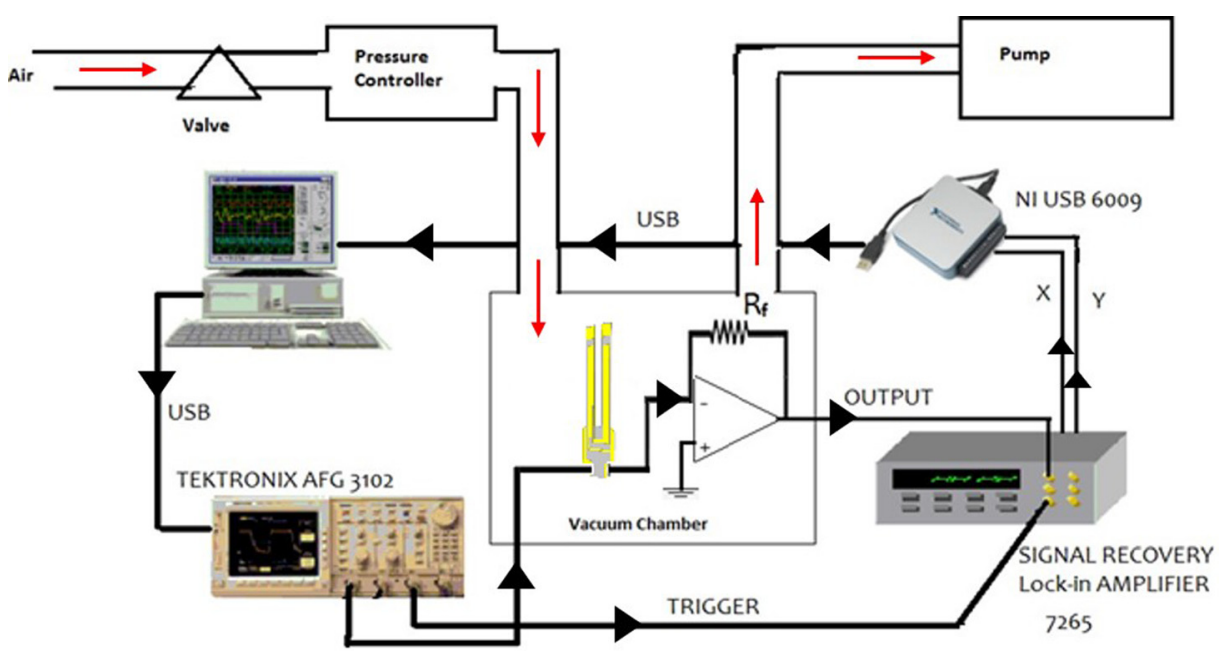

FIG. 12. Schematic of a setup used for electrical characterization of QTFs. The excitation sine voltage is supplied by a high-resolution arbitrary wavefunction generator (AFG), which also provides the reference TTL signal for the lock-in amplifier. The QTF current output is converted to a voltage by means of a transimpedance amplifier. The QTF is mounted inside a vacuum chamber to perform low gas pressure measurements. Red arrows mimic the gas flux direction. 

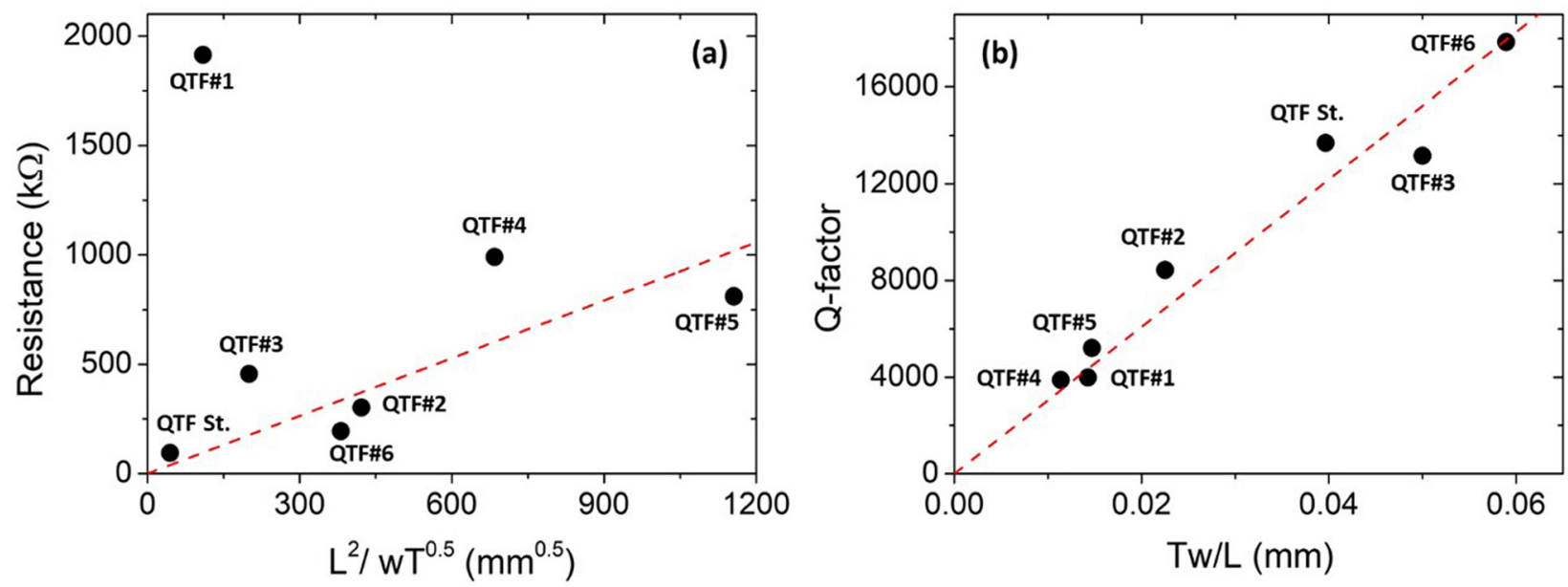

FIG. 13. (a) Dependence of the QTFs resistances versus (• symbols) versus the ratio $L_{p}{ }^{2} / W \cdot T^{1 / 2}$. QTF St. is the standard QTF. The solid curve is the best linear fit considering only the standard QTF (QTF St.) and QTF\#2,3,5,6, using the function $R=n \cdot L_{p}{ }^{2} / W \cdot T^{1 / 2}$, with $n=0.346 \mathrm{k} \Omega / \sqrt{ } \mathrm{mm}$. (b) quality factor $Q(\bullet$ symbols) versus $W T / L_{p}$. The red dashed line is the best linear fit, using the function $Q=m \cdot w \cdot T / L_{p}$, with $m=3.04 \times 10^{5} \mathrm{~mm}^{-1}$. All measurements have been performed at atmospheric pressure.

leading to enhanced amplification of the QEPAS signal. Moreover, at low pressure, the absorption lines are narrow, and the sensor selectivity improves. The extension of the QEPAS technique to the $\mathrm{THz}$ spectral region was initially delayed, mainly due to the difficulty of a proper effective focalization of the $\mathrm{THz}$ laser beam between the prongs of the standard $32 \mathrm{kHz}-\mathrm{QTF}$, since the space between the prongs $(300 \mu \mathrm{m})$ is comparable with the wavelength of the THz radiation. In addition, available $\mathrm{THz}$ sources are characterized by beam profiles with limited spatial quality. The extension of QEPAS in the THz range was first reported with QTF\#6, ${ }^{72,73}$ which has a similar geometry as a commercial $32.7 \mathrm{kHz}-\mathrm{QTF}$, but a prong spacing that is six times wider. A prong design with spacing of $\sim 1 \mathrm{~mm}$ (see Table I) facilitated focalization of the $\mathrm{THz}$ laser beam between the prongs without hitting them. For a first demonstration, a THz QEPAS sensor was designed to detect methanol and to operate at 10 Torr at the resonance frequency of the fundamental mode at $4.245 \mathrm{kHz}$. A cryogenic THz QCL emitting $\sim 40 \mu \mathrm{W}$ at $3.93 \mathrm{THz}(\sim 76.3 \mu \mathrm{m})$ was used as the light source. The oscillation strength of the selected methanol transition is $4.28 \times 10^{-21} \mathrm{~cm}^{-1}$. The QEPAS sensor achieved a detection sensitivity of $7 \mathrm{ppm}$ in $4 \mathrm{~s}$, corresponding to a NNEA $=2 \times 10^{-10} \mathrm{~W} \mathrm{~cm}^{-1} \mathrm{~Hz}^{-1 / 2}$, comparable with the best results of mid-IR QEPAS systems, demonstrating the usefulness of the QEPAS technique in the THz range. In fact, the record NNEA value to-date for QEPAS was obtained in the $\mathrm{THz}$ range, ${ }^{74}$ operating at the same experimental condition of Refs. 72 and 73, but employing QTF\#5. A detection sensitivity of $160 \mathrm{ppb}$ of methanol in $30 \mathrm{~s}$ and a $\mathrm{NNEA}=3.75$ $\times 10^{-11} \mathrm{~cm}^{-1} \mathrm{~W} \mathrm{~Hz}^{-1 / 2}$ were achieved. A photograph of the THz QEPAS setup employed in Ref. 74 is shown in Fig. 14.

The main difference between QTF\#6 and QTF\#5 is the prong spacing. Since for a focused laser beam the decay of the incident acoustic wave pressure on the prong depends critically on the distance between the focused laser beam position and the internal prong surface, the reduction of the prong spacing from $1 \mathrm{~mm}$ (QTF\#6) to $700 \mu \mathrm{m}$ (QTF\#5) led to an improved acousto-electric transduction efficiency and thereby better sensor performance. Further reduction of the prongs spacing is not feasible, because the prongs spacing becomes comparable with the THz QCLs focused beam size $(\sim 500 \mu \mathrm{m})$ and this would lead to a strong increase of the background noise. Another demonstration of THz QEPAS system was reported in Ref. 75 for the detection of hydrogen sulfide $\left(\mathrm{H}_{2} \mathrm{~S}\right)$ detection. The system architecture is based on QTF\#5 and exploited a quantum cascade laser (QCL) emitting $1.1 \mathrm{~mW}$ at a frequency of $2.913 \mathrm{THz}$. The tuning range of the QCL allowed the excitation of a $\mathrm{H}_{2} \mathrm{~S}$ rotational absorption line with a line-strength of $\mathrm{S}=1.13 \times 10^{-22} \mathrm{~cm} /$ mol. $\mathrm{A}_{2} \mathrm{~S}$ detection sensitivity of $30 \mathrm{ppm}$ in $3 \mathrm{~s}$ and $13 \mathrm{ppm}$ for a $30 \mathrm{~s}$ integration time was achieved, corresponding to a $\mathrm{NNEA}=4.4 \times 10^{-10} \mathrm{~W} \cdot \mathrm{cm}^{-1} \cdot \mathrm{Hz}^{-1 / 2}$, which is significantly better than values previously reported for mid-IR and nearIR $\mathrm{H}_{2} \mathrm{~S}$ QEPAS sensors. ${ }^{38,76,77}$

\section{Single-tube micro-resonator systems}

Acoustic micro-resonators are important components to improve the performance of QEPAS sensors. ${ }^{2,14}$ Among the

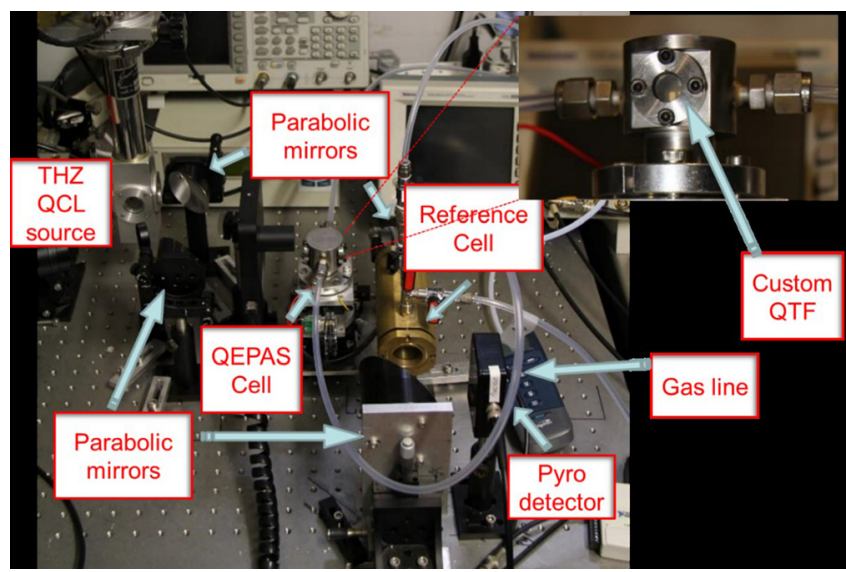

FIG. 14. Photograph of the THz QEPAS based trace gas sensor employed in Ref. 74. The inset on the upper right-hand side of Fig. 14 shows an enlarged view of the QEPAS cell. The upper part of the QTF inside the cell is also visible. 
(a)

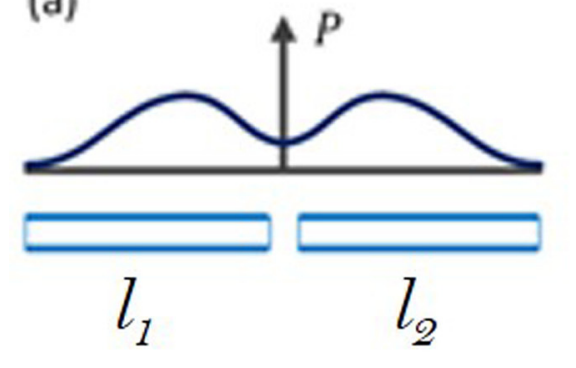

$$
\lambda / 2<l_{1}+l_{2}<\lambda
$$

(b)

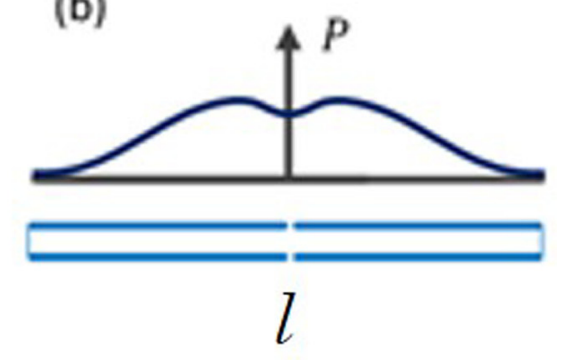

$\lambda / 2<l<\lambda$
FIG. 15. Schematic of the acoustic pressure distributions in two different spectrophone configurations, (a) two tubes separated by a large gap; (b) a single-tube spectrophone configuration. different QEPAS spectrophone configurations, the on-beam one showed the strongest acoustic coupling efficiency between the QTF and the $\mathrm{mR}$, resulting in an optimum signal to noise (SNR) gain factor of $\sim 30$ (Ref. 14) for a standard $32 \mathrm{kHz}-\mathrm{QTF}$. The on-beam spectrophone configuration consists of two stainless steel tubes with the QTF inserted between them. The two half $\mathrm{mR}$ tubes, positioned on the two sides of the QTF, confine the acoustic waves and drive the QTF prongs to vibrate. However, the insertion of the QTF distorts the resonance mode of acoustic standing waves in the $\mathrm{mR}$, as shown schematically in Fig. 15(a), resulting in a reduced pressure field amplification factor and an optimal $\mathrm{mR}$ length falling in the range $\lambda / 2<L<\lambda$, where $\lambda$ is the acoustic wavelength. ${ }^{14,79}$

By decreasing the distance between the two tubes, the distortion of the acoustic mode can be reduced. However, the tubes cannot be located too close to the QTF otherwise damping effects start to dominate. ${ }^{14}$ The optimal distance between $32 \mathrm{kHz}-\mathrm{QTF}$ and tubes was experimentally found and falls in the range of $25 \mu \mathrm{m}-50 \mu \mathrm{m}$. The distortion of the acoustic mode confined within a resonator can be significantly reduced by positioning a single-tube between the prongs of the tuning fork. A pair of slits must be opened on each side of the tube waist, symmetrically in the middle of the $\mathrm{mR}$, where the acoustic pressure antinode and the QTF are located, as schematically shown in Figs. 15(b) and 16. A single tube cannot be employed for the $32 \mathrm{kHz}-\mathrm{QTF}$, since the space between the prongs is only $300 \mu \mathrm{m}$ : a single tube $\sim 1 \mathrm{~cm}$-long and with internal diameter $<300 \mu \mathrm{m}$ would make optical alignment difficult in the near-IR and impractical in the mid-IR and $\mathrm{THz}$ spectral ranges. In contrast, the possibility to operate with custom QTFs with a prong spacing of up to $1 \mathrm{~mm}$ (see Table I) opens the way to the implementation of a single-tube $\mathrm{mR}$ configuration in an on-beam QEPAS spectrophone (SO-QEPAS configuration).

The first demonstration of SO-QEPAS spectrophone was reported in Ref. 80, employing QTF\#2 having a prong spacing of $800 \mu \mathrm{m}$. The optimal single tube $\mathrm{mR}$ geometrical parameters resulted in an internal diameter of $650 \mu \mathrm{m}$ and a length of $38 \mathrm{~mm}$, considering that the acoustic wavelength $\lambda$ for the QTF\#2 operating at $7.2 \mathrm{kHz}$ is $\sim 48 \mathrm{~mm}$. Since the behavior of the single-tube on-beam $\mathrm{mR}$ is similar to that of an ideal one-dimensional acoustic resonator, the optimal single tube length is close to $\lambda / 2$, while in a standard dual-tube
$\mathrm{mR}$ system the total length is close to $\lambda$. The reported optimum full length of the $\mathrm{mR}$ for the traditional two-tubes onbeam spectrophone configuration for QTF\#2 is $46 \mathrm{~mm} .{ }^{78}$ The signal-to-noise amplification factor obtained with the SO-QEPAS configuration resulted in an enhancement factor of $\sim 130$ in comparison with the bare QTF\#2, ${ }^{80}$ while for a standard two-tube $\mathrm{mR}$ the highest reported value is $\sim 40 .{ }^{78} \mathrm{~A}$ more comprehensive study of the impact of the tubes geometry (both single- and dual-tube configuration) on the QEPAS sensor performance employing custom QTF\#2, QTF\#5, and QTF\#6 is reported in Ref. 71.

\section{E. QEPAS with QTF operating in the first overtone flexural mode}

In QEPAS sensing, the resonance frequency of the QTF must be limited below $40 \mathrm{kHz}$ to ensure that the transfer of the excess energy absorbed by the target gas follows efficiently the fast modulation $f$ of the incident laser radiation. ${ }^{2}$ For gases with slow energy relaxation rates (such as $\mathrm{CO}$, $\mathrm{CO}_{2}$, and $\mathrm{NO}$ ), the energy transfer does not efficiently follow the laser modulation at $32 \mathrm{kHz}^{25,26}$ Hence, relaxation promoters (typically $\mathrm{H}_{2} \mathrm{O}$ or $\mathrm{SF}_{6}$ ) are added to the gas sample mixture in order to increase the energy relaxation process.

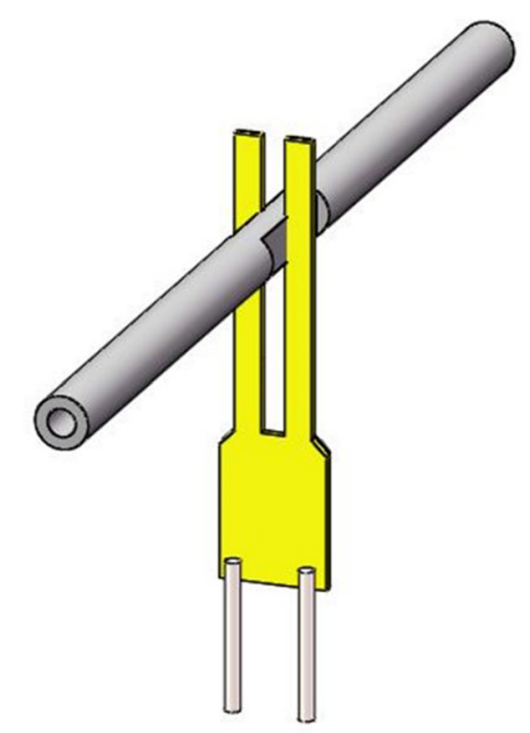

FIG. 16. Schematic of the single-tube micro-resonator configuration. 
Using this approach, enhancements of two orders of magnitude in the QEPAS signal have been reported. ${ }^{2,26,64,82,83}$ This requires accurate measurements of the promoter concentration and additional sensor calibration. A reduction of the operational frequency fulfills the $f \ll 1 / 2 \pi \tau_{T}$ condition for slow-relaxing gases and was one of the main motivations that guided the realization of custom QTFs. However, the first overtone modes frequencies are also reduced with a decrease of the fundamental resonance frequency to $\sim 3 \mathrm{kHz}$. This opened the way to the implementation of QTF overtone flexural modes for QEPAS trace gas sensing. By using Eq. (4), it can be verified that the first overtone mode frequency is $~ 6.2$ times higher than the fundamental mode. For a $32 \mathrm{kHz}-\mathrm{QTF}$, the first overtone mode falls at frequencies $\sim 200 \mathrm{kHz}$ (Ref. 81) and cannot be implemented in a QEPAS based sensor system. The first demonstration of QEPAS system exploiting a QTF operating on the first overtone flexural mode is reported in Ref. 84. In this case, QTF\#5 (having a fundamental resonance frequency at $2.8 \mathrm{kHz}$ and a first overtone mode at $\sim 17 \mathrm{kHz}$ ) was implemented in a QEPAS based sensor system operating in the near-IR spectral range. The selected target gas was water vapor and allowed a comparison of the QEPAS signal using both the fundamental and first overtone QTF flexural modes.

The fundamental and the overtone mode exhibit different quality factors because the loss mechanisms depend on both the resonance frequency and on the vibrational dynamics of the resonance mode. The dominant loss mechanism is vibration damping due to the medium surrounding the vibrating QTF. A resonating QTF prong in a medium can be physically treated as a solid body immersed in an airflow with a small Reynolds number (i.e., in case of laminar flow). The damping dynamics are derived from the Navier-Stokes equation and the continuity equation for incompressible fluids. When the prongs are oscillating at the $n_{-t h}$ natural flexural frequency, the quality factor associated with air damping $Q_{\text {air }}$ can be assumed to be proportional to ${ }^{87}$

$$
Q_{a i r} \propto \frac{4 \pi \rho T W^{2} f_{n}}{3 \pi \mu W+\frac{3}{4} \pi W^{2} \sqrt{4 \pi \rho_{a} \mu f_{n}}},
$$

where $\rho_{a}$ is the air density, $\mu$ is the air viscosity. $Q_{\text {air }}$ increases with the resonance frequency, since the air damping effect decreases with an increasing overtone mode order $n$. If $3 / 4 \pi W^{2} \sqrt{ }\left(4 \pi \rho_{a} \mu f_{n}\right) \gg 3 \pi \mu W$, the $Q_{\text {air }}$ factor can be considered to be quasi-independent of the crystal width $W$, which implies that $f_{n} \gg 4 \mu /\left(\pi \rho_{a} W^{2}\right)$. In standard QTF operating conditions (room temperature and pressure in the range 10-760 Torr), this approximation is valid for overtone modes and leads to the following simplified dependence for the $Q_{\text {air, overtone }}$ based on its geometrical parameters:

$$
Q_{\text {air }, \text { overtone }} \propto \frac{8}{3} \frac{\rho T \sqrt{f_{n}}}{\sqrt{\pi \rho_{a} \mu}} \propto \frac{T^{3 / 2}}{L_{p}} .
$$

For a QTF, the air damping mechanisms are strongly reduced when the QTF vibrates at the overtone mode with respect to the fundamental mode. A second loss mechanism to be considered for a vibrating QTF is support losses. Support losses are due to dissipation of the prong vibrational energy through its support. Hence, support losses are influenced by the vibrational dynamics of the resonance mode. In terms of the $Q$-factor, the contributions of extrinsic and intrinsic loss mechanisms depend on the vibrational dynamics of the selected resonance mode, since each vibrational mode can be characterized by different distributions of its effective mass. ${ }^{85}$ The fundamental in-plane flexural mode can be represented by a single point-mass on the prong tip, while the first overtone can be considered as a system of two-coupled point-mass subsystems, located at two antinodes. The different distribution of the effective mass affects mainly the support losses. Since the dimensions of the support are assumed to be much larger than those of the prong, flexural vibrations can be treated using the single cantilever beam theory. The contribution of the quality factor due to support losses is given by ${ }^{86}$

$$
Q_{\text {sup }} \propto \frac{1}{k_{n}^{2}}\left(\frac{L_{P}}{T}\right)^{3},
$$

where $k_{n}$ is a constant that increases as the mode number increases. Hence, $Q_{s u p}$ is mainly influenced by the ratio between the prong length and its thickness and decreases for higher order modes. For overtone modes, the higher the $L_{p} / T$ ratio, the lower will be the mechanical stress onto the QTF support. The total losses can be evaluated by considering the sum of the different factors causing energy dissipation and hence the overall quality factor for a QTF operating in air can be expressed as

$$
\frac{1}{Q}=\frac{1}{K_{0}}\left(\frac{1}{Q_{\text {sup }}}+\frac{1}{Q_{\text {air }}}\right) .
$$

The factor $K_{0}$ takes into account the fraction of the collected piezoelectric charge with respect to the total generated charge due to the partial coverage of the QTF surface by electric contacts. To reduce the support losses of the overtone modes, the factor $\left(L_{p} / T\right)^{3}$ should be increased [see Eq. (8)], leading to an increase of the air damping losses, affecting negatively the overall quality factor. Thus, even if the support losses increase with the mode number ${ }^{86}$ it is possible to obtain a larger $Q$-factor for overtone modes with respect to the fundamental mode by optimizing the QTF prongs dimensions in order to strongly reduce the air damping mechanisms, which is the dominant loss mechanism.

The first overtone flexural mode can be modeled as 2coupled point-masses, each one positioned at an antinode and oscillating in counter-phase. The two antinodes identify the position of the maximum vibration amplitudes along the prong. A typical calculated vibration profiles of the QTF prongs at the first overtone flexural mode for maxima displacements conditions is shown in Fig. 17 (for QTF\#5) as a function of the distance from the support base for the first overtone mode. The vibrational profile was calculated assuming standing waves propagating along the two QTF prongs. The QEPAS signal is strongly dependent on the position of the laser focusing point along the QTF symmetry axis. When the focusing spot is located at the antinodes 

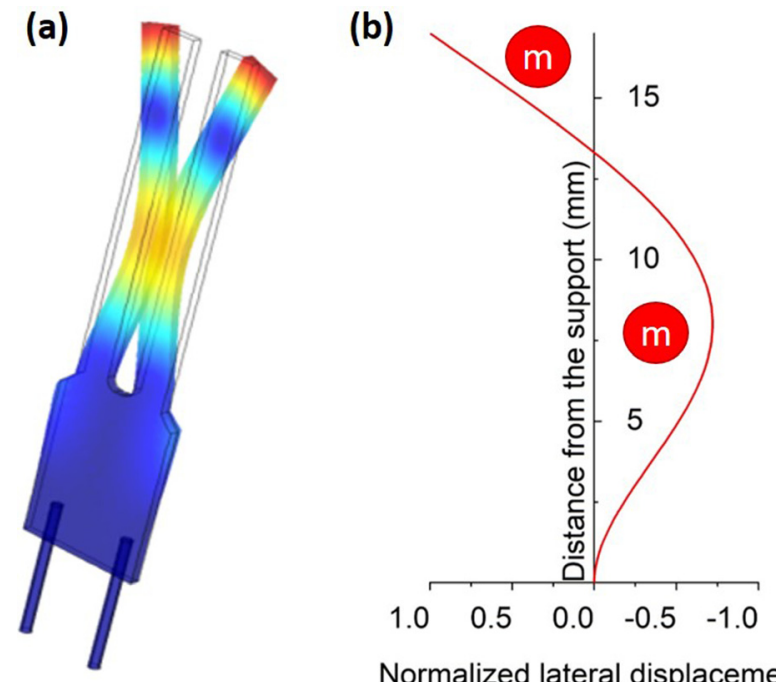

Normalized lateral displacement

FIG. 17. Deformation of the QTF prongs (a) and normalized lateral displacement for the QTF\#5 first overtone mode calculated as a function of distance from the support and normalized with respect to the maximum value occurring at $17 \mathrm{~mm}$ (b). Blue circles represent the point-mass subsystems. Both deformation and vibration profiles have been calculated using Comsol Multiphysics software.

points of the vibration profile, where the maximum vibration amplitude is allowed, the QEPAS signal is maximized.

The optimum laser focusing position along the QTF symmetry axis must be identified for each QTF design by measuring the correlated QEPAS peak signals obtained by slowly scanning step by step the laser focusing position along the QTF symmetry axis. A representative first overtone flexural mode QEPAS scan, obtained for QTF\#4, is shown in Fig. 18. The discrepancy between the QEPAS signal at two antinodes results from the spherical symmetry of the exciting acoustic wave.

The closer to the prong top is the vertical position of the acoustic source, the larger is the fraction of the pressure wave not hitting the QTF prong. This effect downshifts at $0.8 \mathrm{~mm}$ the optimum vertical position of the laser spot for the antinode at the prongs top ${ }^{2}$ and do not influence the lower

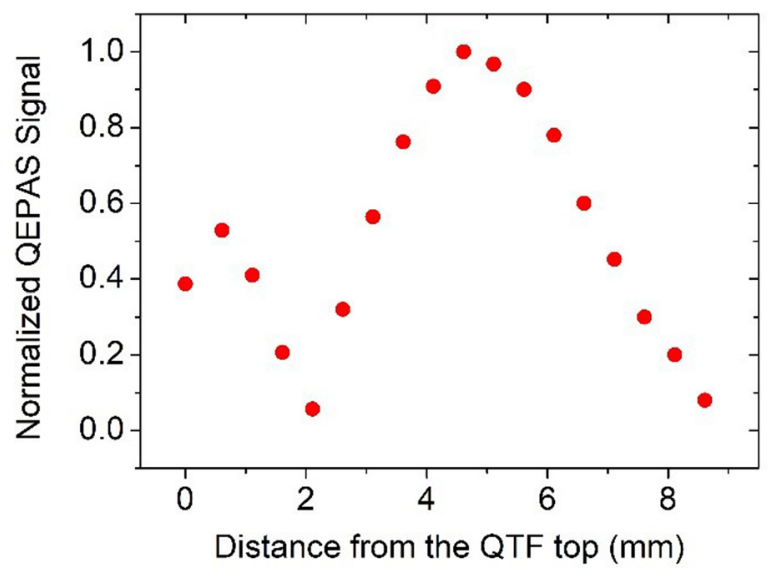

FIG. 18. Normalized QEPAS signal as a function of the beam distance from the QTF support measured for the first overtone mode of QTF\#4 at atmospheric pressure. The laser beam position was scanned along the symmetry axis of the QTF. All data are normalized to the QEPAS highest signal, measured for the first overtone mode at $\mathrm{d}=4.7 \mathrm{~mm}$. antinode at $4.7 \mathrm{~mm}$ that becomes the more efficient one. A similar behavior was observed for other QTFs. ${ }^{52,71,84}$ More detailed investigations of custom QTFs operating in the overtone flexural modes are reported in Refs. 52 and 71 . The resonance frequencies for the overtone mode $\left(f_{l}\right)$ and related $Q$-factors $\left(Q_{1}\right)$ are reported in Table III.

A selection of six custom QTFs were investigated to compare QEPAS signals obtained operating at the QTFs fundamental and first overtone flexural modes. QTF\#2 and QTF\#5 were implemented in QEPAS sensor systems employing a single-mode diode laser as an excitation source, targeting a water absorption line at $7299.43 \mathrm{~cm}^{-1}$ with a line-strength of $1.01 \times 10^{-20} \mathrm{~cm} / \mathrm{mol}^{52}$ whereas QTF\#4 and QTF\#6 were tested using a QCL emitting at $1296.49 \mathrm{~cm}^{-1}$, in resonance with a water absorption line having a linestrength of $1.70 \times 10^{-22} \mathrm{~cm} / \mathrm{mol} .^{71}$ In all cases, the gas samples consisted of air containing a fixed concentration of $1.7 \%$ of water vapor. The focused laser spot was positioned between the two prongs at the vertical positions maximizing the QEPAS signal. In particular, when operating at the QTFs first overtone mode, the lower antinode point was selected. The operating gas pressure and laser modulation amplitude were optimized for each QTF fundamental or first overtone modes. A comparison between the QEPAS spectral scans measured for the two vibrational modes of the investigated QTFs are shown in Fig. 19.

For QTF\#4, QTF\#5, and QTF\#6, the QEPAS peak signal acquired when operating at the QTFs overtone mode is higher than the corresponding one measured using the fundamental mode. These results confirmed that, by optimizing the QTF design, it is possible to favor the first overtone flexural mode operation with respect to the fundamental mode one in terms of QEPAS signal.

\section{F. Single-tube micro-resonator coupled with QTF operating at the first overtone flexural mode}

With respect to the standard $32 \mathrm{kHz}-\mathrm{QTF}$, the realization of QTFs with lower resonance frequency and larger prongs spacing is advantageous in terms of gas target relaxation rates and optical sensor alignment. However, implementing dual-tube or single-tube $\mathrm{mR}$ for QTFs having resonance frequency $<10 \mathrm{kHz}$ becomes challenging. The length of the $\mathrm{mR}$ is correlated with the sound wavelength, given by $\lambda=v / f$, where $v$ is the sound speed ( $343 \mathrm{~m} / \mathrm{s}$ in air). As an example,

TABLE III. Experimental and calculated QTFs physical parameters: resonance frequencies of the first overtone flexural mode $f_{l, v a c}$ (calculated) and $f_{1}^{\left({ }^{\text {exp }}\right)}$ (measured) and related quality factor $Q_{1}$. The operating pressures are also listed.

\begin{tabular}{lcccc}
\hline \hline & $f_{1, \text { vac }}(\mathrm{Hz})$ & $f_{1(\exp )}(\mathrm{Hz})$ & $Q_{1}$ & Pressure (Torr) \\
\hline QTF standard & 200263.1 & 191784.66 & 8500.0 & vacuum \\
QTF \#1 & 86088.96 & 86574.40 & 9464.2 & 760 \\
QTF \#2 & 47456.56 & 41075.90 & 10770.3 & 75 \\
QTF \#3 & 52729.52 & 51009.13 & 15895.2 & 760 \\
QTF \#4 & 21789.04 & 21497.23 & 24533.8 & 75 \\
QTF \#5 & 18245.50 & 17788.95 & 31373.8 & 75 \\
QTF \#6 & 25786.05 & 25413.10 & 9800.0 & 760 \\
\hline \hline
\end{tabular}



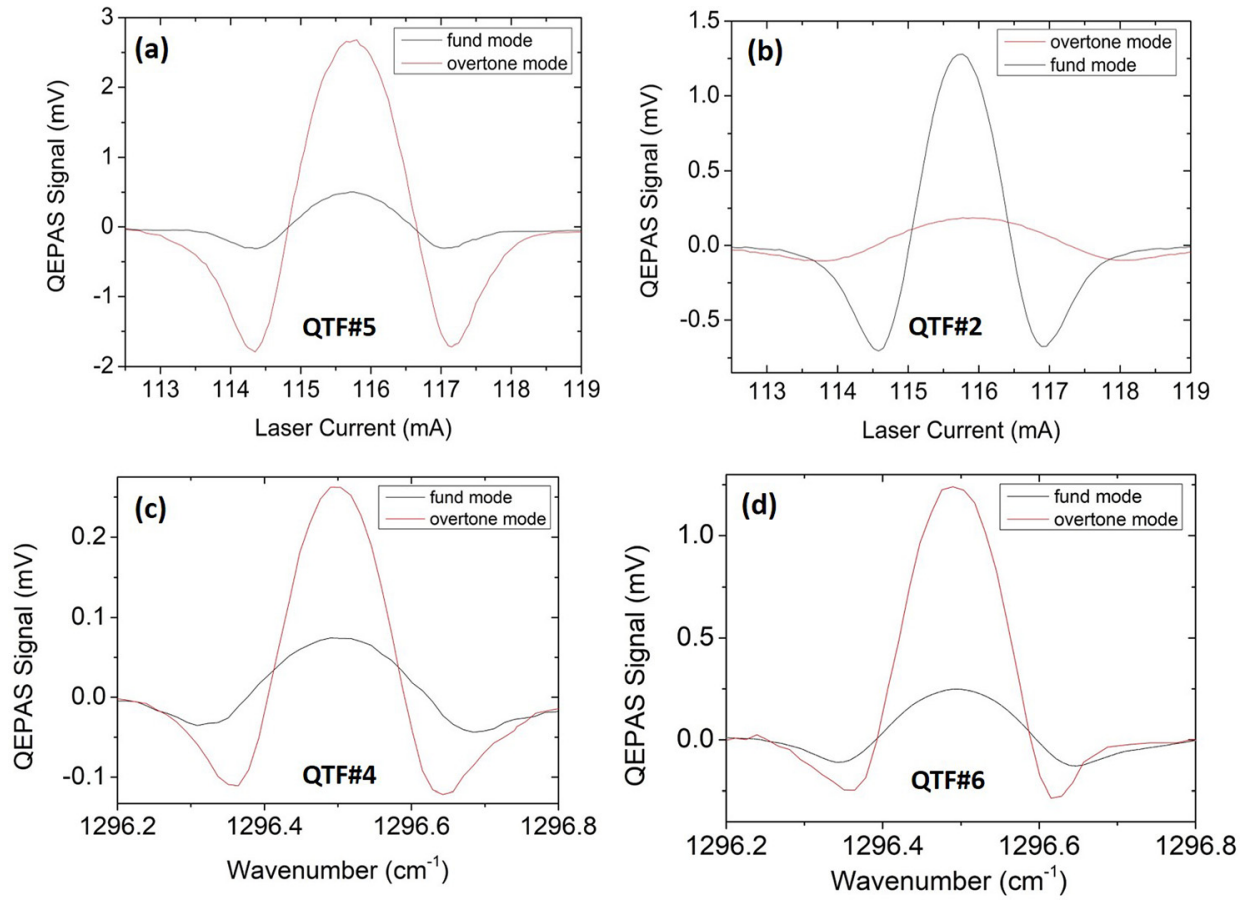

FIG. 19. QEPAS spectral scans of a gas mixture containing air with a $1.7 \%$ water concentration for the fundamental mode (black solid line) and for the first overtone one (red solid line) of the QTF\#5 (a), QTF\#2 (b), QTF\#4 (c), and QTF\#6 (d). QTF\#2 and QTF\#5 were operated at 75 Torr, while QTF\#4 and QTF\#6 were operated at atmospheric pressure. for an $f$ as low as $10 \mathrm{kHz}, \lambda=3.43 \mathrm{~cm}$. As discussed in $\mathrm{Sec}$. III D, the optimal $\mathrm{mR}$ length falls between $\lambda / 2$ and $\lambda$, because of the $\mathrm{mR}$ acoustic coupling with the QTF, closer to $\lambda$ for dual-tube $\mathrm{mR},{ }^{14}$ while closer to $\lambda / 2$ for a single-tube $\mathrm{mR}{ }^{80}$ For QTFs operating at frequencies $<10 \mathrm{kHz}$, the optimal length of the $\mathrm{mR}$ system is several $\mathrm{cm}$. This makes optical alignment challenging when operating with mid-IR and inhibits operations with a $\mathrm{THz}$ laser or excitation sources with limited beam profiles. For example, for QTF\#6 operating at $4.2 \mathrm{kHz}$ and implemented in the first demonstration of a THz QEPAS sensor system, ${ }^{72}$ the optimal single-tube $\mathrm{mR}$ length is $\sim 8 \mathrm{~cm}$. For QTF\#2 operating at $7.2 \mathrm{kHz}$, this length is reduced to $\sim 3.8 \mathrm{~cm}$, still more than 4 times larger than the length of the dual-tube $\mathrm{mR}$ used for the commercial $32 \mathrm{kHz}$ QTF ( 9 mm).

Since the first overtone frequency is $\sim 6.2$ times higher than the fundamental one, operating at the first overtone will require a significantly reduced $\mathrm{mR}$ length, combined with a higher mode quality factor, when the prong sizes are appropriately chosen. The first demonstration of a QEPAS system implementing a QTF operating at the first overtone flexural mode and coupled with a single-tube $\mathrm{mR}$ is reported in Ref. 88. A schematic of the employed setup is shown in Fig. 20.

A single-mode continuous-wave distributed feedback (DFB) laser emitting at $1.37 \mu \mathrm{m}$ was employed as the excitation source to generate photoacoustic signals, targeting a water vapor absorption line. QTF\#5 was implemented for this demonstration and the support losses were significantly reduced due to the optimization of geometrical ratio $L_{p} / T$, providing a more than $2 \times$ higher $Q$-factor for the overtone mode with respect to the fundamental mode. ${ }^{52,84}$ The maximum signal amplitude was obtained for a single-tube length of $14.5 \mathrm{~mm}$, larger than $\lambda / 2(9.5 \mathrm{~mm})$, proving that the first harmonic acoustic standing waves in the $\mathrm{mR}$ were partially distorted by the two slits present in the resonator. The highest signal amplitude obtained is $\sim 50$ times larger than that measured using a bare QTF operating in the first overtone resonance mode and $\sim 380$ times higher than that obtained with the bare QTF operating in fundamental resonance mode. The achieved sensitivity enhancement factor is $\sim 13$

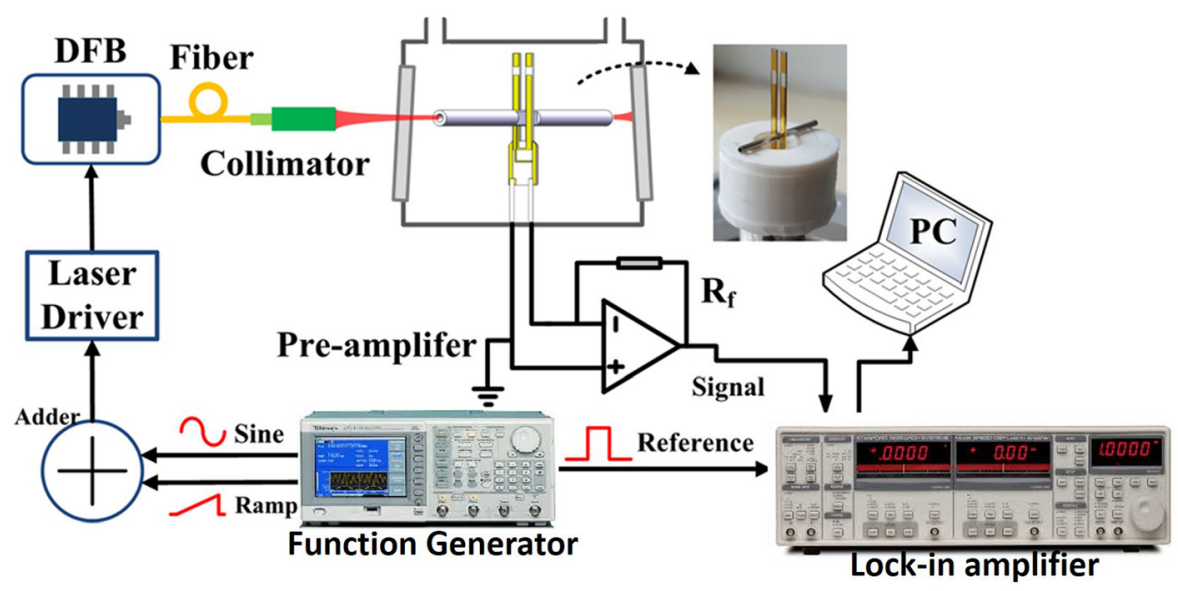

FIG. 20. Schematic of the experimental setup. DFB: distributed feedback laser; PC: personal computer; $\mathrm{R}_{\mathrm{f}}$ : feedback resistance. 


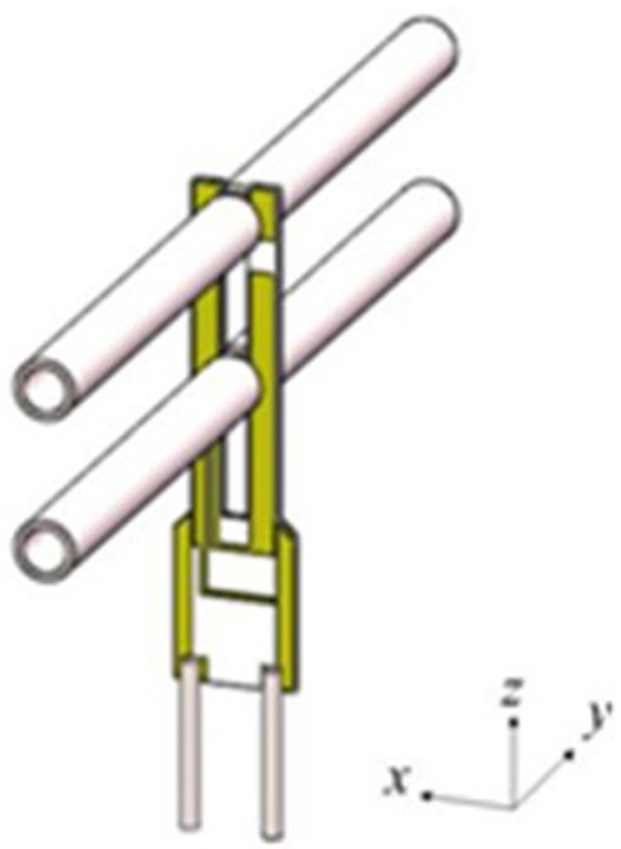

FIG. 21. Schematic of the DAE-QEPAS spectrophone.

times higher than that attained by a conventional QEPAS spectrophone based on a commercial $32 \mathrm{kHz}-\mathrm{QTF} .{ }^{14}$

\section{G. Double antinode excited quartz-enhanced photoacoustic spectrophone}

Different from a fundamental flexural mode, the first overtone mode oscillation shows two antinodes, oscillating in counter-phase. Hence, it is possible to excite two resonance antinode points simultaneously by using two laser beams if their phase shift is properly adjusted. The first demonstration of a double-antinode excited quartz-enhanced photoacoustic spectroscopy (DAE-QEPAS) system was reported in Ref. 89, implementing QTF\#5 and a dual-tube $\mathrm{mR}$ system. The schematic of the double antinode excited QEPAS (DAE-QEPAS) spectrophone is depicted in Fig. 21.

The QTF is positioned between the two mRs tubes to probe the acoustic vibration excited in the gas contained inside the tubes and the two mRs are positioned at heights corresponding to the lower and higher QTF first overtone resonance antinode points. The schematic of the DAEQEPAS setup is shown in Fig. 22.

A pigtailed distributed feedback (DFB) laser emitting at $1.37 \mu \mathrm{m}$ was employed to generate the photoacoustic signal, by exciting a $\mathrm{H}_{2} \mathrm{O}$ absorption line located at $7306.75 \mathrm{~cm}^{-1}$ with a line intensity of $1.8 \times 10^{-20} \mathrm{~cm} / \mathrm{mol}$. The PZT phase compensator was used to adjust the phase between the two laser beams passing through the two mRs in order to maximize the QEPAS signal. The phase shift between the two antinode points is $\sim 180^{\circ}$. The laser beam passed twice through the lower $\mathrm{mR}$ and once through the upper $\mathrm{mR}$, exciting the two QTF resonance antinodes simultaneously with a compensated phase. The largest QEPAS SNR was obtained employing $8.5 \mathrm{~mm}$-long tubes for the two $\mathrm{mRs}$. The achieved signal amplitude was $\sim 3$ times higher than that obtained with standard on-beam QEPAS, $\sim 100$ times higher than that obtained with a bare QTF operating at the first overtone mode and $\sim 500$ times higher when operating at the fundamental flexural mode resonance. A comparison of the best results achieved with the different on-beam spectrophone configuration reported to date is shown in Table IV.

The minimum $\mathrm{mR}$ length for custom QTFs demonstrated so far is $14.5 \mathrm{~mm}$ comparable with the standard $32 \mathrm{kHz}-\mathrm{QTF}$ $\mathrm{mR}$ length $(\sim 10 \mathrm{~mm})$. Further improvement of the SNR gain factor can be achieved either by assembling two single-tube $\mathrm{mRs}$ exploiting the SO-QEPAS configuration or by designing and implementing an octupole configuration for the QTF gold

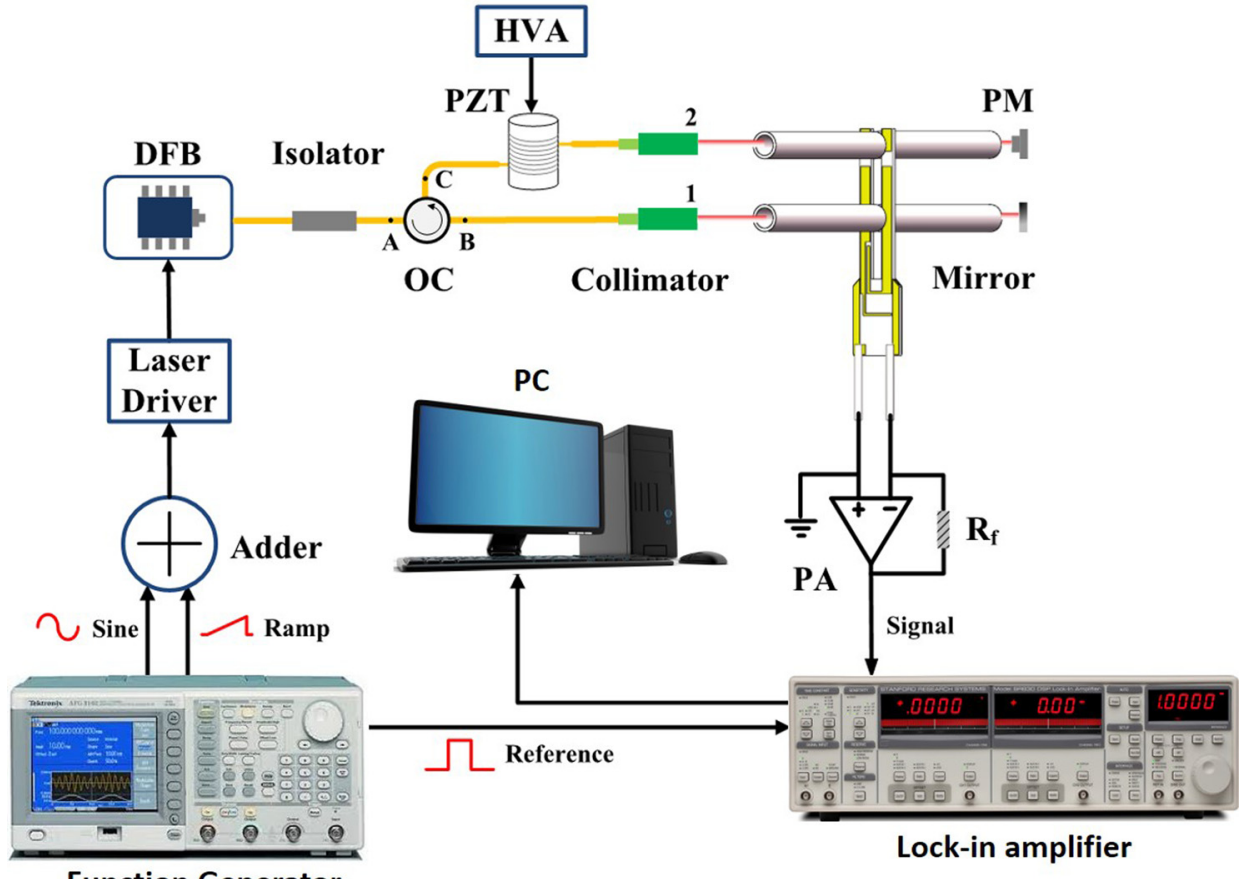

FIG. 22. Schematic of the DAEQEPAS setup. DFB: distributed feedback diode laser; OC: optical circulator; PZT: piezoelectric transducer; HVA: high voltage amplifier; PM: power meter; PA: pre-amplifier; PC: personal computer; $\mathrm{R}_{\mathrm{f}}$ : feedback resistance. 
TABLE IV. Comparison of on-beam QEPAS Spectrophone configurations. OD: other tube diameter and ID: inner tube diameter.

\begin{tabular}{|c|c|c|c|c|c|c|c|}
\hline QTF & Configuration & QTF\# & $\mathrm{OD}(\mathrm{mm})$ & $\mathrm{ID}(\mathrm{mm})$ & $\mathrm{mR}$ length $(\mathrm{mm})$ & Gain factor & References \\
\hline \multirow[t]{5}{*}{ Custom } & Bare QTF & 2 & & & & 1 & 80 \\
\hline & Two-tubes & 2 & 1.5 & 1.3 & 46 & 40 & 78 \\
\hline & Single-tube & 2 & 0.9 & 0.65 & 38 & 130 & 80 \\
\hline & Single-tube + overtone & 2 & 0.98 & 0.62 & 14.5 & 380 & 88 \\
\hline & Double antinode + overtone (dual-tube) & 5 & 1.58 & 1.3 & 19 & 500 & 89 \\
\hline \multirow[t]{2}{*}{ Standard } & Bare QTF & Stand. & & & & 1 & 2 \\
\hline & On-beam & Stand. & 1.24 & 0.8 & 10.0 & 30 & 14 \\
\hline
\end{tabular}

pattern in order to increase the charge collection efficiency of the first overtone flexural mode resonance. The possibility to increase the space between the prongs, potentially up to few $\mathrm{mm}$ will allow to employ $\mathrm{mR}$ tubes with a large ID, thereby simplifying optical alignment and making the QEPAS spectrophone less sensitive to external vibrations in future field applications such as sensing on trains or on-drones.

\section{H. Simultaneous dual-gas detection}

Multi-gas on-line detection is a topic of considerable interest in recent years because of its important applications in environmental monitoring, medical diagnosis, industrial process control, agriculture and food industries, and pollution monitoring. In several cases, like isotope concentration ratios, to retrieve the actual ratio value, the concentration measurements of two isotopes must be performed simultaneously. This is also the case when it is required to control the relative concentration of two different gas target in a matrix that rapidly changes with time, as in agricultural applications or from sample to sample, as in breath analysis (e.g., NO and $\mathrm{H}_{2} \mathrm{O}$ ). With a standard QEPAS approach, simultaneous gas detection is not feasible since the force sensing based QTF cannot recognize the molecular source of an induced acoustic wave. Even when a single laser source is able to separate two absorption features referred to two different gas species, there is a delay in time in the measurements of two different target gas concentrations, since the laser source has to be tuned from one absorption line to the other absorption line.

However, due to the reduction in the fundamental resonance frequency achieved with custom QTFs, the first overtone flexural mode becomes available for QEPAS operation, allowing the simultaneous excitation of the QTF both on the fundamental and first overtone modes, using the same or two different laser sources. Recently, this approach was employed in the first demonstration of simultaneous dual-gas sensing, based on a QTF frequency division multiplexing (FDM) technique. ${ }^{90}$ The QTF in a dual-gas QEPAS sensor is excited simultaneously at the fundamental and first overtone flexural modes by two independently modulated lasers. The two target gases are detected via demodulation of the custom QTF piezoelectric signal at the fundamental frequency $f_{0}$ and the first overtone frequency $f_{1}$, respectively, by means of two lock-in amplifiers. The schematic of the developed dual-gas QEPAS system is shown in Fig. 23(a). QTF\#5 was enclosed inside a gas cell and two laser beams were focused on the QTF plane. The focused beams were positioned near to the antinode point for the fundamental flexural mode [blue dot in Fig. 23(b)] and close to the first overtone flexural mode lower antinode point [red dot in Fig. 23(b)], respectively. The QTF was connected to a custom transimpedance

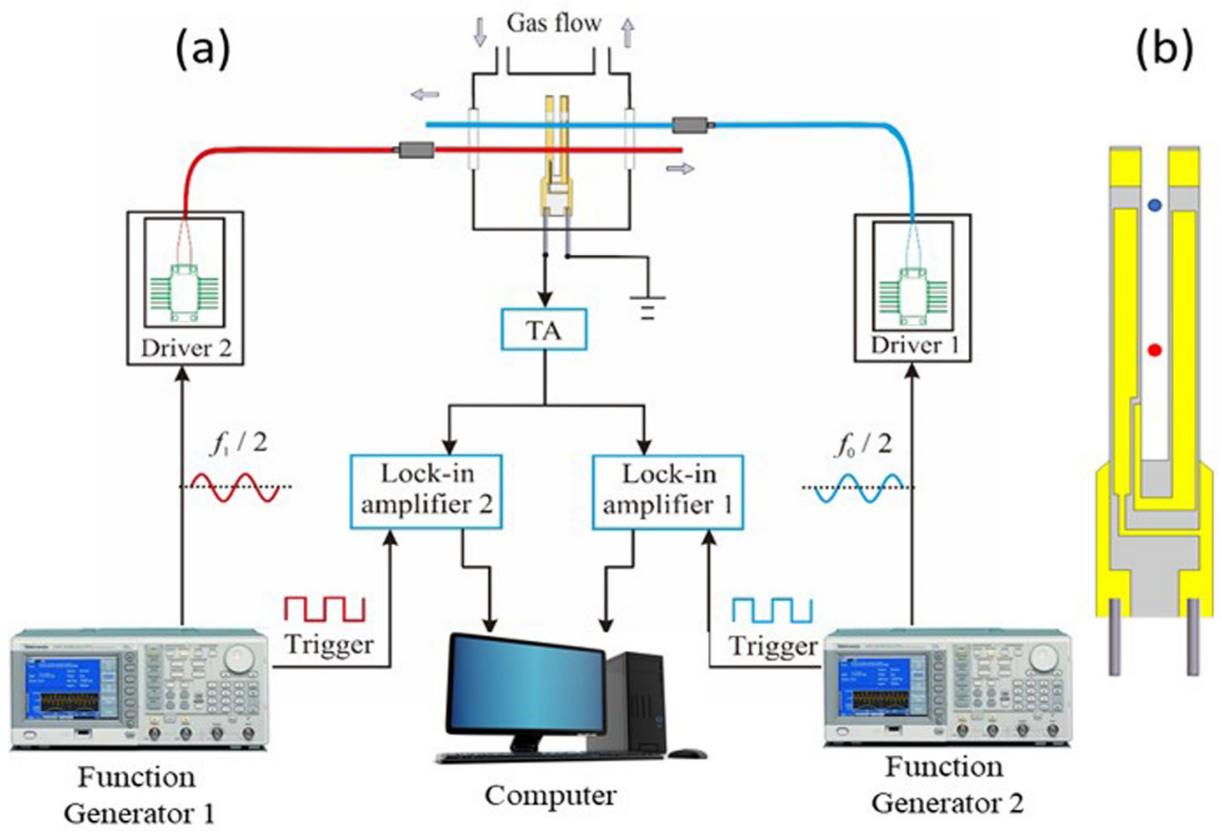

FIG. 23. (a) Schematic of a dual-gas QEPAS sensor system based on a QTF combined vibration at fundamental and first overtone modes. TA: transimpedance amplifier. (b) QTF\#5 with two focused laser beam spots represented by the red and blue dots. 
amplifier, which delivered the amplified signal to two lockin amplifiers for wavelength modulation detection.

A preliminary test with both laser sources tuned to the same wavelength at $1368.6 \mathrm{~nm}$, resonant with a $\mathrm{H}_{2} \mathrm{O}$ absorption line was performed to verify that there are no crosstalking effects between the fundamental and the first overtone flexural mode QEPAS signals, when the QTF was operated in the combined vibrations. No interference effects and no excess noise were observed when the QTF operates in combined vibrational motion with respect to the pure fundamental or first overtone flexural mode. The capability of the QEPAS sensor to perform simultaneous dual-gas spectral detection was demonstrated by implementing a DFB laser source targeting an acetylene $\left(\mathrm{C}_{2} \mathrm{H}_{2}\right)$ absorption line located at $6541.96 \mathrm{~cm}^{-1}$ with a line-strength of $3.5 \times 10^{-20} \mathrm{~cm} \cdot \mathrm{mol}^{-1}$ (Ref. 91) and a diode laser targeting a $\mathrm{H}_{2} \mathrm{O}$ absorption line. The QEPAS spectra of both $\mathrm{H}_{2} \mathrm{O}$ and $\mathrm{C}_{2} \mathrm{H}_{2}$ were acquired continuously without signal cross-talking. The sensor performance in terms of NNEA coefficient for $\mathrm{H}_{2} \mathrm{O}$ and $\mathrm{C}_{2} \mathrm{H}_{2}$ are $9.12 \times 10^{-7} \mathrm{~cm}^{-1} \cdot \mathrm{W} / \mathrm{Hz}^{1 / 2}$ and $1.51 \times 10^{-7} \mathrm{~cm}^{-1} \cdot \mathrm{W} / \mathrm{Hz}_{1 / 2}$, respectively. The difference in performance is due to the different QEPAS response of QTF\#5 for the fundamental vibrational mode with respect to the first overtone mode, which is $\sim 6$ times higher for the latter. This difference can be adjusted by optimizing the QTF prongs geometry.

Applications of a dual-gas QEPAS system include detection of gases with slow energy relaxation rates (such as NO, $\mathrm{CO}$, and $\mathrm{CH}_{4}$ ). As mentioned previously, to enhance the QEPAS signal, relaxation promoters (typically $\mathrm{H}_{2} \mathrm{O}$ or $\mathrm{SF}_{6}$ ) are added to the gas mixture and are crucial to control their concentration levels. Thereby, the additional detection channel can be devoted to monitoring the relaxation promoter concentration and using this information to normalize the slowrelaxing gas QEPAS signal amplitude. ${ }^{82}$ Further improvements of dual-gas QEPAS sensors performances can be achieved by adding dual- or single-tube acoustic micro-resonators to enhance the generated photo-acoustic wave intensity.

\section{CONCLUSIONS AND OUTLOOK}

This review has focused on recent advances in the QEPAS sensing technique for the detection and monitoring of trace gas species. Progress to-date in terms of custom QTF development, spectrophone configurations, and new approaches for QEPAS sensing were reviewed. Compared with a $32 \mathrm{kHz}-\mathrm{QTF}$ configured with a dual tube $\mathrm{mR}$ system, representing the standard for QEPAS sensors, the first generation of custom $\mathrm{QTFs}^{67}$ results in better sensing performance, as demonstrated in Ref. 80, where QTF\#2 coupled with a single-tube $\mathrm{mR}$ provided a 4 times higher SNR factor and a $\sim 1.5$ improvement factor of the NNEA $\left(1.21 \times 10^{-8}\right.$ $\mathrm{cm}^{-1} \cdot \mathrm{W} / \sqrt{\mathrm{Hz}}$ for QTF\#5 as compared to $1.8 \times 10^{-8}$ $\mathrm{cm}^{-1} \cdot \mathrm{W} / \sqrt{\mathrm{Hz}}$ for the $32 \mathrm{kHz}-\mathrm{QTF}$ ), at the same operating conditions. Furthermore, the possibility to increase the prongs spacing makes the optical alignment less critical, especially with laser sources having limited spatial beam quality. Custom QTFs allowed the extension of the QEPAS technique in the $\mathrm{THz}$ range, where, due to the fast rotationalvibrational relaxation rates, the QEPAS technique is more efficient, as demonstrated by the record NNEA factor achieved so far $\left[3.75 \times 10^{-11} \mathrm{~cm}^{-1} \mathrm{~W} \cdot \mathrm{Hz}^{-1 / 2}\right.$ (Ref. 74)] The reduction of the fundamental flexural mode resonances in custom QTFs opened the way to the exploitation of the first overtone mode for QEPAS sensing. This leads to the demonstration of new approaches such as the double antinode excited QEPAS and the first demonstration of simultaneous dual-gas detection with an optical sensor due to the possibility to excite simultaneously the QTF at the fundamental and the first overtone flexural modes.

In conclusion, several novel approaches of QEPAS based trace gas sensors have been demonstrated, potentially improving the sensitivity, size, robustness, and cost, making these sensors mature for real-world applications, including environmental monitoring [ $\mathrm{CO}, \mathrm{CO}_{2}, \mathrm{NO}, \mathrm{CH}_{4}, \mathrm{H}_{2} \mathrm{CO}$, $\mathrm{C}_{2} \mathrm{HF}_{5}, \mathrm{~N}_{2} \mathrm{O}, \mathrm{NO}_{2}$ (Refs. 25, 26, 29, 48, 54, 55, and 92-95)], industrial applications $\left[\mathrm{HCl}, \mathrm{CH}_{4}, \mathrm{C}_{2} \mathrm{H}_{6}, \mathrm{H}_{2} \mathrm{~S}, \mathrm{SF}_{6} \mathrm{C}_{2} \mathrm{H}_{2}\right.$ (Refs. 16, 38, and 96-98)], agricultural applications $\left[\mathrm{C}_{2} \mathrm{H}_{4}\right.$, $\mathrm{CH}_{4}, \mathrm{~N}_{2} \mathrm{O}$ (Refs. 94 and 99)], and breath analysis (NO, CO, $\left.\mathrm{NH}_{3}, \mathrm{C}_{2} \mathrm{H}_{6}, \mathrm{H}_{2} \mathrm{~S}, \mathrm{HCN}\right){ }^{25,26,38,96,100,101}$ Thanks to the compactness and robustness of QEPAS based sensors modules, other interesting real world chemical sensing applications such as sensing-on-drones and on rail vehicles can be envisaged.

\section{ACKNOWLEDGMENTS}

The authors from Dipartimento Interateneo di Fisica di Bari acknowledge financial support from THORLABS $\mathrm{GmbH}$, within PolySense, a joint-research laboratory. The author from Shanxi University acknowledges financial support from the National Natural Science Foundation of China (Nos. 61622503 and 61575113) and the Outstanding Innovative Teams of Higher Learning Institutions of Shanxi. Frank K. Tittel acknowledges the support by the Welch Foundation (Grant No. R4925U).

${ }^{1}$ J. Hodgkinson and R. P. Tatam, Meas. Sci. Technol. 24, 012004 (2013).

${ }^{2}$ P. Patimisco, G. Scamarcio, F. K. Tittel, and V. Spagnolo, Sensors 14, 6165 (2014).

${ }^{3}$ G. Eranna, B. C. Joshi, D. P. Runthala, and R. P. Gupta, Crit. Rev. Solid State Mater. Sci. 29, 111-188 (2004).

${ }^{4}$ D. Kohl, J. Phys. D: Appl. Phys. 34, R125-R149 (2001).

${ }^{5}$ C. Wang, L. Yin, L. Zhang, D. Xiang, and R. Gao, Sensors 10, 2088-2106 (2010).

${ }^{6}$ P. R. Fortes, J. F. S. Petruci, and I. M. Raimundo, Optical Gas Sensors for Exhaled Breath Analysis (SPIE Press, Bellingham, Washington, USA, 2017).

${ }^{7}$ E. Bakker and M. Telting-Diaz, Anal. Chem. 74, 2781-2800 (2002).

${ }^{8}$ I. Galli, S. Bartalini, S. Borri, P. Cancio, D. Mazzotti, P. de Natale, and

G. Giusfredi, Phys. Rev. Lett. 107, 270802 (2011).

${ }^{9}$ L. Xiong, W. Bai, F. Chen, X. Zhao, F. Yu, and G. J. Diebold, Proc. Natl. Acad. Sci. U.S.A. 114, 7246-7249 (2017).

${ }^{10}$ A. Elia, P. M. Lugarà, C. di Franco, and V. Spagnolo, Sensors 9, 9616-9628 (2009).

${ }^{11}$ W. H. Flygare, Acc. Chem. Res. 1(4), 121-127 (1968).

${ }^{12}$ A. A. Kosterev, Y. A. Bakhirkin, R. F. Curl, and F. K. Tittel, Opt. Lett. 27, 1902-1904 (2002).

${ }^{13}$ A. A. Kosterev, F. K. Tittel, D. Serebryakov, A. Malinovsky, and A. Morozov, Rev. Sci. Instrum. 76, 043105 (2005).

${ }^{14}$ L. Dong, A. A. Kosterev, D. Thomazy, and F. K. Tittel, Appl. Phys. B 100, 627-635 (2010).

${ }^{15}$ M. Jahjah, S. Belahsene, L. Nahle, M. Fischer, J. Koeth, Y. Rouillard, and A. Vicet, Opt. Lett. 37, 2502-2504 (2012). 
${ }^{16}$ V. Spagnolo, P. Patimisco, S. Borri, G. Scamarcio, B. E. Bernacki, and J. Kriesel, Opt. Lett. 37, 4461-4463 (2012).

${ }^{17}$ T. N. Ba, M. Triki, G. Desbrosses, and A. Vicet, Rev. Sci. Instrum. 86, 023111 (2015).

${ }^{18}$ K. Liu, X. Guo, H. Yi, W. Chen, W. Zhang, and X. Gao, Opt. Lett. 34, 1594-1596 (2009).

${ }^{19}$ S. Böttger, M. Köhring, U. Willer, and W. Schade, Appl. Phys. B 113, 227-232 (2013).

${ }^{20}$ J. P. Waclawek, H. Moser, and B. Lendl, Opt. Express 24, 6559-6571 (2016).

${ }^{21}$ M. Lassen, L. Lamard, Y. Feng, A. Peremans, and J. C. Petersen, Opt. Lett. 41, 4118-4121 (2016).

${ }^{22} \mathrm{H}$. Yi, K. Liu, W. Chen, T. Tan, L. Wang, and X. Gao, Opt. Lett. 36, 481-483 (2011).

${ }^{23}$ K. Liu, H. Yi, A. A. Kosterev, W. Chen, L. Dong, L. Wang, T. Tan, W. Zhang, F. K. Tittel, and X. Gao, Rev. Sci. Instrum. 81, 103103 (2010).

${ }^{24}$ Z. Wang, Q. Wang, J. Y. L. Ching, J. C. Y. Wu, G. Zhang, and W. Ren, Sens. Actuators, B 246, 710-715 (2017).

${ }^{25}$ V. Spagnolo, A. A. Kosterev, L. Dong, R. Lewicki, and F. K. Tittel, Appl. Phys. B 100, 125-130 (2010).

${ }^{26}$ L. Dong, V. Spagnolo, R. Lewicki, and F. K. Tittel, Opt. Express 19, 24037-24045 (2011).

${ }^{27}$ R. Lewicki, G. Wysocki, A. A. Kosterev, and F. K. Tittel, Opt. Express 15, 7357-7366 (2007).

${ }^{28}$ A. A. Kosterev, P. R. Buerki, L. Dong, M. Reed, T. Day, and F. K. Tittel, Appl. Phys. B 100, 173-180 (2010).

${ }^{29}$ P. Patimisco, A. Sampaolo, Y. Bidaux, A. Bismuto, M. Schott, J. Jiang, A. Muller, J. Faist, F. K. Tittel, and V. Spagnolo, Opt. Express 24, 25943-25954 (2016).

${ }^{30}$ P. Patimisco, V. Spagnolo, M. S. Vitiello, A. Tredicucci, G. Scamarcio, C. M. Bledt, and J. A. Harrington, Appl. Phys. B 108, 255-260 (2012).

${ }^{31}$ P. Patimisco, V. Spagnolo, M. S. Vitiello, A. Tredicucci, G. Scamarcio, C. M. Bledt, and J. A. Harrington, Sensors 13, 1329-1340 (2013).

${ }^{32}$ A. Sampaolo, P. Patimisco, J. M. Kriesel, F. K. Tittel, G. Scamarcio, and V. Spagnolo, Opt. Express 23, 195-204 (2015).

${ }^{33}$ P. Patimisco, A. Sampaolo, L. Mihai, M. Giglio, J. Kriesel, D. Sporea, G. Scamarcio, F. K. Tittel, and V. Spagnolo, Sensors 16, 533 (2016).

${ }^{34}$ N. Hô, M. C. Phillips, H. Qiao, P. J. Allen, K. Krishnaswami, B. J. Riley, T. L. Myers, and N. C. Anheier, Opt. Lett. 31, 1860-1862 (2006).

${ }^{35}$ C. Tsay, Y. Zha, and C. B. Arnold, Opt. Express 18, 26744-26753 (2010)

${ }^{36}$ C. Caillaud, G. Renversez, L. Brilland, D. Mechin, L. Calvez, J.-L. Adam, and J. Troles, Materials 7(9), 6120-6129 (2014).

${ }^{37}$ V. Spagnolo, P. Patimisco, S. Borri, G. Scamarcio, B. E. Bernacki, and J. Kriesel, Appl. Phys. B 112, 25-33 (2013).

${ }^{38}$ M. Siciliani de Cumis, S. Viciani, S. Borri, P. Patimisco, A. Sampaolo, G. Scamarcio, P. De Natale, F. D'Amato, and V. Spagnolo, Opt. Express 22(23), 28222-28231 (2014).

${ }^{39}$ M. Köhring, U. Willer, S. Böttger, A. Pohlkötter, and W. Schade, IEEE J. Sel. Top. Quantum Electron. 18, 1566-1572 (2012).

${ }^{40}$ Y. Ma, G. Yu, J. Zhang, X. Yu, and R. Sun, J. Opt. 17, 055401 (2015).

${ }^{41}$ Y. Cao, W. Jin, L. H. Ho, and Z. Liu, Opt. Lett. 37, 214-216 (2012).

${ }^{42}$ Y. Cao, W. Jin, and L. H. Ho, Proc. SPIE 8351, 835121 (2012).

${ }^{43}$ Z. Li, Z. Wang, C. Wang, and W. Ren, Appl. Phys. B 122, 147 (2016).

${ }^{44}$ Z. Li, Z. Wang, Y. Qi, W. Jin, and W. Ren, Sens. Actuators, B 248, 1023-1028 (2017)

${ }^{45}$ V. Spagnolo, L. Dong, A. A. Kosterev, D. Thomazy, J. Doty, and F. K. Tittel, Opt. Lett. 36, 460-462 (2011).

${ }^{46}$ V. Spagnolo, L. Dong, A. A. Kosterev, D. Thomazy, J. Doty, and F. K. Tittel, Appl. Phys. B 103, 735-742 (2011).

${ }^{47}$ V. Spagnolo, L. Dong, A. A. Kosterev, and F. K. Tittel, Opt. Express 20, 3401-3407 (2012).

${ }^{48}$ H. Zheng, L. Dong, X. Yin, X. Liu, H. Wu, L. Zhang, W. Ma, W. Yin, and S. Jia, Sens. Actuators, B 208, 173-179 (2015).

${ }^{49}$ H. Wu, L. Dong, X. Liu, H. Zheng, X. Yin, W. Ma, L. Zhang, W. Yin, and S. Jia, Sensors 15, 26743-26755 (2015).

${ }^{50}$ H. Wu, L. Dong, H. Zheng, X. Liu, X. Yin, W. Ma, L. Zhang, W. Yin, S. Jia, and F. K. Tittel, Sens. Actuators, B 221, 666-672 (2015).

${ }^{51}$ H. Wu, L. Dong, H. Zheng, Y. Yu, W. Ma, L. Zhang, W. Yin, L. Xiao, S. Jia, and F. K. Tittel, Nat. Commun. 8, 15331 (2017).

${ }^{52}$ F. K. Tittel, A. Sampaolo, P. Patimisco, L. Dong, A. Geras, T. Starecki, and V. Spagnolo, Opt. Express 24, A682 (2016).

${ }^{53}$ L. Dong, R. Lewicki, K. Liu, P. R. Buerki, M. J. Weida, and F. K. Tittel, Appl. Phys. B 107, 275-283 (2012).
${ }^{54}$ S. Borri, P. Patimisco, I. Galli, D. Mazzotti, G. Giusfredi, N. Akikusa, M. Yamanishi, G. Scamarcio, P. De Natale, and V. Spagnolo, Appl. Phys. Lett. 104, 091114 (2014).

${ }^{55}$ P. Patimisco, S. Borri, I. Galli, D. Mazzotti, G. Giusfredi, N. Akikusa, M. Yamanishi, G. Scamarcio, P. De Natale, and V. Spagnolo, Analyst 140, 736-743 (2015).

${ }^{56}$ J. Wojtas, A. Gluszek, A. Hudzikowski, and F. K. Tittel, Sensors 17, 513 (2017).

${ }^{57}$ P. Patimisco, A. Sampaolo, F. K. Tittel, and V. Spagnolo, Sens. Actuators, A 267, 70-75 (2017).

${ }^{58}$ N. Petra, J. Zweck, A. A. Kosterev, S. E. Minkoff, and D. Thomazy, Appl. Phys. B 94, 673-680 (2009).

${ }^{59}$ H. Wu, L. Dong, W. Ren, W. Yin, W. Ma, L. Zhang, S. Jia, and F. K. Tittel, Sens. Actuators, B 206, 364-370 (2015).

${ }^{60}$ L. Dong, H. Wu, H. Zheng, Y. Liu, X. Liu, W. Jiang, L. Zhang, W. Ma, W. Ren, W. Yin, S. Jia, and F. K. Tittel, Opt. Lett. 39, 2479-2482 (2014).

${ }^{61}$ Y. Ma, X. Yu, G. Yu, X. Li, J. Zhang, D. Chen, R. Sun, and F. K. Tittel, Appl. Phys. Lett. 107, 021106 (2015).

${ }^{62}$ H. Zheng, X. Yin, L. Dong, H. Wu, X. Liu, W. Ma, L. Zhang, W. Yin, and S. Jia, J. Spectrosc. 2015, 218413.

${ }^{63}$ W. Griffith, J. Appl. Phys. 21, 1319 (1950).

${ }^{64}$ A. A. Kosterev, Y. A. Bakhirkin, R. F. Curl, and F. K. Tittel, Appl. Phys. B 80, 133 (2005).

${ }^{65}$ J. Li, W. Chen, and B. Yu, Appl. Sprectrosc. Rev. 46, 440-471 (2011).

${ }^{66}$ F. R. Blom, S. Bouwstra, M. Elwenspoek, and J. H. J. Fluitman, J. Vac. Sci. Technol. B 10, 19 (1992).

${ }^{67}$ P. Patimisco, A. Sampaolo, L. Dong, M. Giglio, G. Scamarcio, F. K. Tittel, and V. Spagnolo, Sens. Actuators, B 227, 539-546 (2016).

${ }^{68}$ M. Hirata, K. Kokubun, M. Ono, and K. Nakayama, J. Vac. Sci. Technol. A 3, 1742 (1985)

${ }^{69}$ M. Christen, Sens. Actuators 4, 555-554 (1983).

${ }^{70} \mathrm{~W}$. Zhang and K. Turner, Sens. Actuators, A 134, 594-599 (2007).

${ }^{71}$ P. Patimisco, A. Sampaolo, H. Zheng, L. Dong, F. K. Tittel, and V. Spagnolo, Adv. Phys. X 2, 169-187 (2016).

${ }^{72}$ S. Borri, P. Patimisco, A. Sampaolo, H. E. Beere, D. A. Ritchie, M. S. Vitiello, G. Scamarcio, and V. Spagnolo, Appl. Phys. Lett. 103, 021105 (2013).

${ }^{73}$ P. Patimisco, S. Borri, A. Sampaolo, H. E. Beere, D. A. Ritchie, M. S. Vitiello, G. Scamarcio, and V. Spagnolo, Analyst 139, 2079-2087 (2014).

${ }^{74}$ A. Sampaolo, P. Patimisco, M. Giglio, M. S. Vitiello, H. E. Beere, D. A. Ritchie, G. Scamarcio, F. K. Tittel, and V. Spagnolo, Sensors 16, 439 (2016).

${ }^{75}$ V. Spagnolo, P. Patimisco, R. Pennetta, A. Sampaolo, G. Scamarcio, M. S. Vitiello, and F. K. Tittel, Opt. Express 23, 7574-7582 (2015).

${ }^{76}$ A. A. Kosterev, L. Dong, D. Thomazy, F. K. Tittel, and S. Overby, Appl. Phys. B 101, 649 (2010).

${ }^{77}$ S. Viciani, M. Siciliani de Cumis, S. Borri, P. Patimisco, A. Sampaolo, G. Scamarcio, P. De Natale, F. D'Amato, and V. Spagnolo, Appl. Phys. B 119, 21-27 (2015).

${ }^{78}$ H. Wu, A. Sampaolo, L. Dong, P. Patimisco, X. Liu, H. Zheng, X. Yin, W. Ma, L. Zhang, W. Yin, V. Spagnolo, S. Jia, and F. K. Tittel, Appl. Phys. Lett. 107, 111104 (2015).

${ }^{79}$ D. V. Serebryakov, I. V. Morozov, A. A. Kosterev, and V. S. Letokhov, Quantum Electron. 40, 167 (2010).

${ }^{80}$ H. Zheng, L. Dong, A. Sampaolo, H. Wu, P. Patimisco, X. Yin, W. Ma, L. Zhang, W. Yin, V. Spagnolo, S. Jia, and F. K. Tittel, Opt. Lett. 41, 978-981 (2016).

${ }^{81}$ J. M. Friedt and É. Carry, Am. J. Phys. 75, 415 (2007).

${ }^{82}$ X. Yin, L. Dong, H. Zheng, X. Liu, H. Wu, Y. Yang, W. Ma, L. Zhang, W. Yin, L. Xiao, and S. Jia, Sensors 16, 162 (2016).

${ }^{83}$ M. Mordmüller, M. Köhring, W. Schade, and U. Willer, Appl. Phys. B 119, 111-118 (2015)

${ }^{84}$ A. Sampaolo, P. Patimisco, L. Dong, A. Geras, G. Scamarcio, T. Starecki, F. K. Tittel, and V. Spagnolo, Appl. Phys. Lett. 107, 231102 (2015).

${ }^{85}$ R. R. Archer, N. H. Cook, S. H. Crandall, N. C. Dahl, F. A. McClintock, E. Rabinowicz, and G. S. Reichenbach, An Introduction to the Mechanics of Solids (McGraw-Hill, New York, 1959).

${ }^{86}$ Z. Hao, A. Erbil, and F. Ayazi, Sens. Actuators, A 109, 156 (2003).

${ }^{87}$ H. Hosaka, K. Itao, and S. Kuroda, Sens. Actuators, A 49, 87-95 (1995).

${ }^{88}$ H. Zheng, L. Dong, A. Sampaolo, P. Patimisco, W. Ma, L. Zhang, W. Yin, L. Xiao, V. Spagnolo, S. Jia, and F. K. Tittel, Appl. Phys. Lett. 109, 111103 (2016) 
${ }^{89}$ H. Zheng, L. Dong, P. Patimisco, H. Wu, A. Sampaolo, X. Yin, S. Li, W. Ma, L. Zhang, W. Yin, L. Xiao, V. Spagnolo, S. Jia, and F. K. Tittel, Appl. Phys. Lett. 110, 021110 (2017).

${ }^{90}$ H. Wu, X. Yin, L. Dong, K. Pei, A. Sampaolo, P. Patimisco, H. Zheng, W. Ma, L. Zhang, W. Yin, L. Xiao, V. Spagnolo, S. Jia, and F. K. Tittel, Appl. Phys. Lett. 110, 121104 (2017).

${ }^{91}$ See http://www.hitran.com for HITRAN Database.

${ }^{92}$ Y. Ma, R. Lewicki, M. Razeghi, and F. K. Tittel, Opt. Express 21, 1008-1019 (2013).

${ }^{93}$ G. Wysocki, A. A. Kosterev, and F. K. Tittel, Appl. Phys. B 85, 301-306 (2006)

${ }^{94}$ M. Jahjah, W. Kiang, N. P. Sanchez, W. Ren, P. Patimisco, V. Spagnolo, S. C. Herndon, R. J. Griffin, and F. K. Tittel, Opt. Lett. 39, 957 (2014).
${ }^{95}$ F. K. Tittel, G. Wysocki, A. A. Kosterev, and Y. A. Bakhirkin, MidInfrared Coherent Sources and Applications (Springer, Houten, The Netherlands, 2007).

${ }^{96}$ A. K. Y. Ngai, S. T. Persijn, I. D. Linsday, A. A. Kosterev, P. Grob, C. J. Lee, S. M. Cristescu, and F. K. Tittel, Appl. Phys. B 89, 123-128 (2007).

${ }^{97}$ Y. Ma, Y. He, X. Yu, C. Chen, R. Sun, and F. K. Tittel, Sens. Actuators, B 233, 388-393 (2016)

${ }^{98}$ Y. Ma, Y. He, L. Zhang, X. Yu, J. Zhang, R. Sun, and F. K. Tittel, Appl. Phys. Lett. 110, 031107 (2017)

${ }^{99}$ Z. Wang, J. Geng, and W. Ren, Appl. Spectrosc. 71, 1834-1841 (2017).

${ }^{100}$ A. A. Kosterev, T. S. Mosely, and F. K. Tittel, Appl. Phys. B 85, 295-300 (2006).

${ }^{101}$ L. Dong, J. Wright, B. Peters, B. A. Ferguson, F. K. Tittel, and S. McWhorter, Appl. Phys. B 107, 459-467 (2012). 